\title{
Experimental Analysis of Space-Bounded Schedulers
}

\author{
HARSHA VARDHAN SIMHADRI, Lawrence Berkeley National Lab \\ GUY E. BLELLOCH, Carnegie Mellon University \\ JEREMY T. FINEMAN, Georgetown University \\ PHILLIP B. GIBBONS, Intel Labs Pittsburgh \\ AAPO KYROLA, Carnegie Mellon University
}

\begin{abstract}
The running time of nested parallel programs on shared-memory machines depends in significant part on how well the scheduler mapping the program to the machine is optimized for the organization of caches and processor cores on the machine. Recent work proposed "space-bounded schedulers" for scheduling such programs on the multilevel cache hierarchies of current machines. The main benefit of this class of schedulers is that they provably preserve locality of the program at every level in the hierarchy, which can result in fewer cache misses and better use of bandwidth than the popular work-stealing scheduler. On the other hand, compared to work stealing, space-bounded schedulers are inferior at load balancing and may have greater scheduling overheads, raising the question as to the relative effectiveness of the two schedulers in practice.

In this article, we provide the first experimental study aimed at addressing this question. To facilitate this study, we built a flexible experimental framework with separate interfaces for programs and schedulers. This enables a head-to-head comparison of the relative strengths of schedulers in terms of running times and cache miss counts across a range of benchmarks. (The framework is validated by comparisons with the Intel ${ }^{\circledR}$ Cilk ${ }^{\mathrm{TM}}$ Plus work-stealing scheduler.) We present experimental results on a 32-core Xeon ${ }^{\circledR} 7560$ comparing work stealing, hierarchy-minded work stealing, and two variants of space-bounded schedulers on both divide-and-conquer microbenchmarks and some popular algorithmic kernels. Our results indicate that space-bounded schedulers reduce the number of L3 cache misses compared to work-stealing schedulers by $25 \%$ to $65 \%$ for most of the benchmarks, but incur up to $27 \%$ additional scheduler and load-imbalance overhead. Only for memory-intensive benchmarks can the reduction in cache misses overcome the added overhead, resulting in up to a $25 \%$ improvement in running time for synthetic benchmarks and about $20 \%$ improvement for algorithmic kernels. We also quantify runtime improvements varying the available bandwidth per core (the "bandwidth gap") and show up to 50\% improvements in the running times of kernels as this gap increases fourfold. As part of our study, we generalize prior definitions of space-bounded schedulers to allow for more practical variants (while still preserving their guarantees) and explore implementation tradeoffs.
\end{abstract}

Categories and Subject Descriptors: D.3.4 [Processors]: Runtime Environments; D.1.3 [Concurrent Programming]: Parallel Programming

General Terms: Scheduling, Algorithms, Performance

This work is supported in part by the National Science Foundation under grant numbers CCF-1018188, CCF-1314633, CCF-1314590, the Intel Science and Technology Center for Cloud Computing (ISTC-CC), the U.S. Department of Energy, Office of Science, Office of Advanced Scientific Computing Research, Applied Mathematics and Computer Science programs under contract No. DE-AC02-05CH11231, and a Facebook Graduate Fellowship.

Authors' addresses: H. V. Simhadri, Computer Science Department, Lawrence Berkeley National Lab, 1 Cyclotron Rd, Berkeley CA 94720; email: harshas@lbl.gov; G. E. Blelloch, P. B. Gibbons, and A. Kyrola, Computer Science Department, Carnegie Mellon University, 5000 Forbes Ave, Pittsburgh PA 15213; emails: \{guyb, gibbons, akyrola\}@cs.cmu.edu; J. T. Fineman, Department of Computer Science, 329A Saint Mary's Hall, Georgetown University, 37th and O Streets, NW, Washington D.C. 20057-1232; email: jfineman@cs. georgetown.edu.

Permission to make digital or hard copies of part or all of this work for personal or classroom use is granted without fee provided that copies are not made or distributed for profit or commercial advantage and that copies show this notice on the first page or initial screen of a display along with the full citation. Copyrights for components of this work owned by others than ACM must be honored. Abstracting with credit is permitted. To copy otherwise, to republish, to post on servers, to redistribute to lists, or to use any component of this work in other works requires prior specific permission and/or a fee. Permissions may be requested from Publications Dept., ACM, Inc., 2 Penn Plaza, Suite 701, New York, NY 10121-0701 USA, fax +1 (212) 869-0481, or permissions@acm.org.

(c) 2016 ACM 2329-4949/2016/06-ART8 $\$ 15.00$

DOI: http://dx.doi.org/10.1145/2938389 
Additional Key Words and Phrases: Thread schedulers, space-bounded schedulers, work stealing, cache misses, multicores, memory bandwidth

\section{ACM Reference Format:}

Harsha Vardhan Simhadri, Guy E. Blelloch, Jeremy T. Fineman, Phillip B. Gibbons, and Aapo Kyrola. 2016. Experimental analysis of space-bounded schedulers. ACM Trans. Parallel Comput. 3, 1, Article 8 (June 2016), 27 pages.

DOI: http://dx.doi.org/10.1145/2938389

\section{INTRODUCTION}

Writing nested parallel programs using fork-join primitives on top of a unified memory space is an elegant and productive way to program parallel machines. Nested parallel programs are portable, sufficiently expressive for many algorithmic problems [Shun et al. 2012; Blelloch et al. 2012], relatively easy to analyze [Blumofe and Leiserson 1999; Blelloch et al. 2010, 2011], and supported by many programming languages including OpenMP [OpenMP Architecture Review Board 2008], Cilk++ [Leiserson 2010], Intel ${ }^{\circledR}$ TBB [Intel 2013a], Java Fork-Join [Lea 2000], and Microsoft TPL [Microsoft 2013]. The unified memory address space hides from programmers the complexity of managing a diverse set of physical memory components like RAM and caches. Processor cores can access memory locations without explicitly specifying their physical location. Beneath this interface, however, the real cost of accessing a memory address from a core can vary widely, depending on where in the machine's cache/memory hierarchy the data resides at time of access. Runtime thread schedulers can play a large role in determining this cost, by optimizing the timing and placement of program tasks for effective use of the machine's caches.

Machine Models and Schedulers. Robust schedulers for mapping nested parallel programs to machines with certain kinds of simple cache organizations such as single-level shared and private caches have been proposed. They work well both in theory [Blumofe et al. 1996; Blumofe and Leiserson 1999; Blelloch et al. 1999] and in practice [Frigo et al. 1998; Narlikar 2002]. Among these, the work-stealing scheduler is particularly appealing for private caches because of its simplicity and low overheads, and is widely deployed in various runtime systems such as Cilk++. The PDF scheduler [Blelloch et al. 1999] is suited for shared caches, and practical versions of this scheduler have been studied [Narlikar 2002]. The cost of these schedulers in terms of cache misses or running times can be bounded by the locality cost of the programs as measured in certain abstract program-centric cost models [Blumofe et al. 1996; Acar et al. 2002; Blelloch et al. 2011, 2013; Simhadri 2013].

However, modern parallel machines have multiple levels of cache, with each cache shared among a subset of cores (e.g., see Figure 1(a)). A parallel memory hierarchy $(\mathrm{PMH})$ as represented by a tree of caches [Alpern et al. 1993] (Figure 1(b)) is a reasonably accurate and tractable model for such machines [Fatahalian et al. 2006; Chowdhury et al. 2010, 2013; Blelloch et al. 2011]. Because previously studied schedulers for simple machine models may not be optimal for these complex machines, recent work has proposed a variety of hierarchy-aware schedulers [Fatahalian et al. 2006; Chowdhury et al. 2010, 2013; Quintin and Wagner 2010; Blelloch et al. 2011] for use on such machines. For example, hierarchy-aware work-stealing schedulers such as the priority work-stealing (PWS) and hierarchial work-stealing (HWS) schedulers [Quintin and Wagner 2010] have been proposed, but no theoretical bounds are known.

To address this gap, space-bounded schedulers [Chowdhury et al. 2010, 2013] have been proposed and analyzed. In a space-bounded scheduler, it is assumed that the computation has a nested (hierarchical) structure. The goal of a space-bounded scheduler is to match the space taken by a subcomputation to the space available on some level 


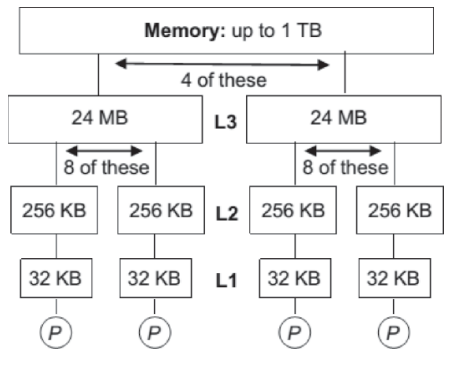

(a) 32-core Xeon (B) 7560

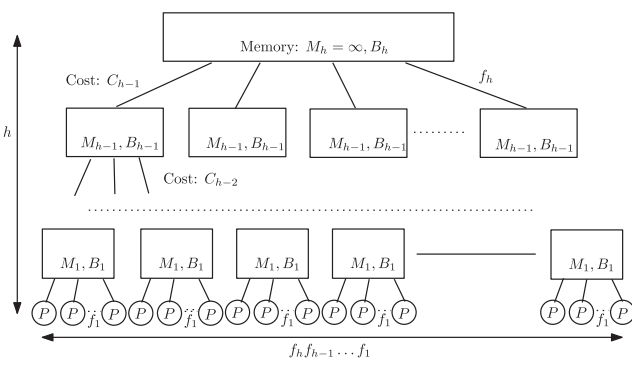

(b) PMH model of Alpern et al. [1993]

Fig. 1. Memory hierarchy of a current-generation architecture from Intel ${ }^{\circledR}$, plus an example abstract parallel hierarchy model. Each cache (rectangle) is shared by all cores (circles) in its subtree. The parameters of the PMH model are explained in Section 2.

of the machine hierarchy. For example, if a machine has some number of shared caches each with $m$-bytes of memory and $k$ cores, then once a subcomputation fits within $m$ bytes, the scheduler can assign it to one of these caches. The subcomputation is then said to be pinned to that shared cache and all of its subcomputations must run on the $k$ cores belonging to it. This ensures that all data is shared within the cache. For this to work, the scheduler must know (a good upper bound on) a subcomputation's space requirement when it starts. In this article, we assume each function in a computation is annotated with the size of its memory footprint, which is passed to the scheduler (see Appendix A for an illustration). This size is typically computed during execution from function arguments such as the number of elements in a subarray. Note that these space annotations are program centric: that is, a property of a computation, not a machine-the computation can be oblivious to the size of the caches and hence is portable across machines. Therefore, the space requirement of each subcomputation can be estimated by hand calculation for most algorithmic computations. A program profiler could also be used to estimate space.

Under certain conditions, such space-bounded schedulers can guarantee good bounds on cache misses at every level of the hierarchy and running time in terms of some intuitive program-centric metrics. Chowdhury et al. [2010] (updated as a journal article in Chowdhury et al. [2013]) presented such schedulers with strong asymptotic bounds on cache misses and runtime for highly balanced computations. Our follow-on work [Blelloch et al. 2011] presented slightly generalized schedulers that obtain similarly strong bounds for unbalanced computations.

Our Results: The First Experimental Study of Space-Bounded Schedulers. While space-bounded schedulers have good theoretical guarantees on the PMH model, there has been no experimental study to suggest that these (asymptotic) guarantees translate into good performance on real machines with multilevel caches. Existing analyses of these schedulers ignore the overhead costs of the scheduler itself and account only for the program runtime. Intuitively, given the low overheads and highly adaptive load balancing of work stealing in practice, space-bounded schedulers would seem to be inferior on both accounts, but superior in terms of cache misses. This raises the question as to the relative effectiveness of the two types of schedulers in practice.

This article presents the first experimental study aimed at addressing this question through a head-to-head comparison of work-stealing and space-bounded schedulers. To facilitate a fair comparison of the schedulers on various benchmarks, it is necessary to have a framework that provides separate modular interfaces for writing portable nested parallel programs and specifying schedulers. The framework should be lightweight and flexible, provide fine-grained timers, and enable access to various hardware counters 
for cache misses, clock cycles, and so forth. Prior scheduler frameworks, such as the Sequoia framework [Fatahalian et al. 2006], which implements a scheduler that closely resembles a space-bounded scheduler, fall short of these goals by (1) forcing a program to specify the specific sizes of the levels of the hierarchy it is intended for, making it nonportable, and (2) lacking the flexibility to readily support work stealing or its variants.

This article describes a scheduler framework that we designed and implemented, which achieves these goals. To specify a (nested-parallel) program in the framework, the programmer uses a Fork-Join primitive (and a Parallel-For built on top of ForkJoin). To specify the scheduler, one needs to implement just three primitives describing the management of tasks at Fork and Join points: add, get, and done (semantics in Section 3). Any scheduler can be described in this framework as long as the schedule does not require the preemption of sequential segments of the program. A simple workstealing scheduler, for example, can be described with only tens of lines of code in this framework. Furthermore, in this framework, program tasks are completely managed by the schedulers, allowing them full control of the execution.

The framework enables a head-to-head comparison of the relative strengths of schedulers in terms of running times and cache miss counts across a range of benchmarks. (The framework is validated by comparisons with the commercial Cilk ${ }^{\mathrm{TM}}$ Plus workstealing scheduler.) We present experimental results on a 32 -core Intel ${ }^{\circledR}$ Nehalem series Xeon ${ }^{\circledR} 7560$ multicore with three levels of cache. As depicted in Figure 1(a), each L3 cache is shared (among the eight cores on a socket), while the L1 and L2 caches are exclusive to cores. We compare four schedulers-work-stealing, priority work-stealing (PWS) [Quintin and Wagner 2010], and two variants of space-bounded schedulers—on both divide-and-conquer microbenchmarks (scan based and gather based) and popular algorithmic kernels such as quicksort, sample sort, quad trees, quickhull, matrix multiplication, triangular solver, and Cholesky factorization.

Our results indicate that space-bounded schedulers reduce the number of L3 cache misses compared to work-stealing schedulers by $25 \%$ to $65 \%$ for most of the benchmarks, while incurring up to $27 \%$ additional overhead. For memory-intensive benchmarks, the reduction in cache misses overcomes the added overhead, resulting in up to a $25 \%$ improvement in running time for synthetic benchmarks and about $20 \%$ improvement for algorithmic kernels. To better understand how the widening gap between processing power (cores) and memory bandwidth impacts scheduler performance, we quantify runtime improvements over a fourfold range in the available bandwidth per core and show further improvements in the running times of kernels (up to 50\%) as the bandwidth gap increases.

Contributions. The contributions of this article are as follows:

-A modular framework for describing schedulers, machines as tree of caches, and nested parallel programs (Section 3). The framework is equipped with timers and counters. Schedulers that are expected to work well on tree of cache models such as space-bounded schedulers and certain work-stealing schedulers are implemented.

- The first experimental study of space-bounded schedulers and the first head-tohead comparison with work-stealing schedulers (Section 5). On a common multicore machine configuration (four sockets, 32 cores, three levels of caches), we quantify the reduction in L3 cache misses incurred by space-bounded schedulers relative to both work-stealing variants on synthetic and nonsynthetic benchmarks. On bandwidthbound benchmarks, an improvement in cache misses translates to improvement in running times, although some of the improvement is eroded by the greater overhead of the space-bounded scheduler. We also explore implementation tradeoffs in spacebounded schedulers. 

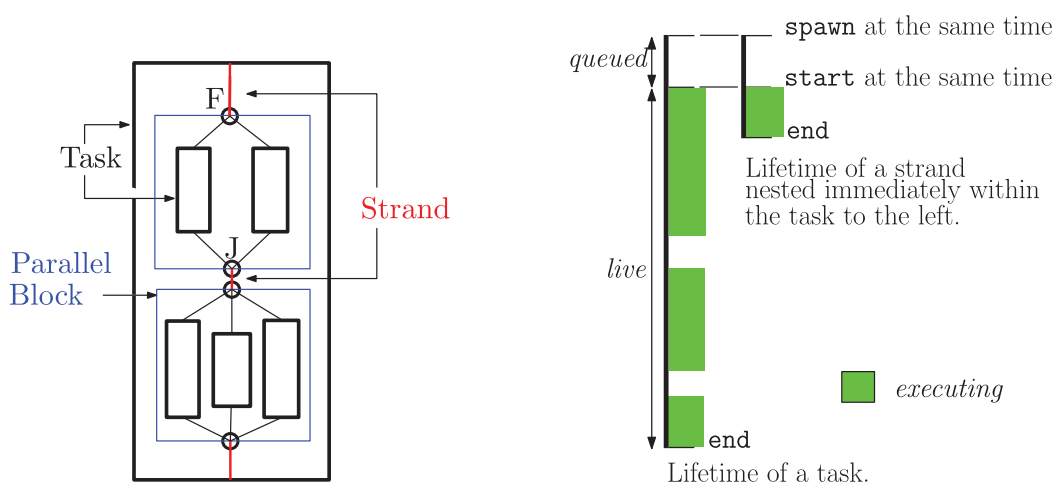

Fig. 2. (Left) Decomposing the computation: tasks, strands, and parallel blocks. $F$ and $J$ are corresponding fork and join points. (Right) Timeline of a task and its first strand, showing the difference between being live and execution.

Note that in order to produce efficient implementations, we provide a slightly broader definition (Section 4) of space-bounded schedulers than in previous work [Chowdhury et al. 2010, 2013; Blelloch et al. 2011]. The key difference is in the scheduling of sequential subcomputations, called strands. Specifically, we introduce a new parameter (constant $\mu$ ) that limits the maximum space footprint of a sequential strand, thereby allowing multiple "large" strands to be scheduled simultaneously on a cache. Without this optimization, we found that space-bounded schedulers do not expose enough parallelism to effectively load-balance the computation. We also generalize the space annotations themselves, allowing strands to be annotated directly rather than inheriting their space from a parent task. This latter change has a minor impact on the definition of space-bounded schedulers, but it may yield better programmability. We describe two variants of space-bounded schedulers, highlighting the engineering details that allow for low overhead.

\section{DEFINITIONS}

We start with a recursive definition of nested parallel computations and use it to define what constitutes a schedule. We will then define the parallel memory hierarchy model-a machine model that reasonably accurately represents shared-memory parallel machines with deep memory hierarchies. This terminology will be used later to define schedulers for the PMH model.

Computation Model, Tasks, and Strands. We consider computations with nested parallelism, allowing arbitrary dynamic nesting of fork-join constructs including parallel loops, but no other synchronizations. This corresponds to the class of algorithms with series-parallel dependence graphs (see Figure 2 (left)).

Nested parallel computations can be decomposed into "tasks," "parallel blocks," and "strands" recursively as follows. As a base case, a strand is a serial sequence of instructions not containing any parallel constructs or subtasks. A task is formed by serially composing $k \geq 1$ strands interleaved with $(k-1)$ "parallel blocks," denoted by $\mathrm{t}=\ell_{1} ; \mathrm{b}_{1} ; \ldots ; \ell_{k}$. A parallel block is formed by composing in parallel one or more tasks with a fork point before all of them and a join point after (denoted by $\mathrm{b}=\mathrm{t}_{1}\left\|\mathrm{t}_{2}\right\| \ldots \| \mathrm{t}_{k}$ ). A parallel block can be, for example, a parallel loop or some constant number of recursive calls. The top-level computation is a task.

A strand always ends in a fork or a join. The strand that immediately follows a parallel block with fork $F$ and join $J$ is referred to as the continuation of $F$ or $J$. If a strand $\ell$ ends in a fork $F, \ell$ immediately precedes the first strand in the tasks 
that constitute the parallel block forked by $F$. If $\ell$ ends in a join $J$, it immediately precedes the continuation of $J$. We define the logical precedence relation between strands in a task as the transitive closure of the immediate precedence relation defined earlier. They induce a partial ordering on the strands in the top-level task. The first strand in any task logically precedes every other strand in it; the last strand in a task is logically preceded by every strand in it. Further, the logical precedence relation induces a directed series-parallel graph on the strands of a task with the first and the last strands as the source and the sink terminals. We refer to this as the dependence graph or the DAG of a task.

We use the notation $L(t)$ to indicate all strands that are recursively included in a task. For every strand $\ell$, there exists a task $\mathrm{t}(\ell)$ such that $\ell$ is nested immediately inside $\mathrm{t}(\ell)$. We call this the task of strand $\ell$.

Our computation model assumes all strands share a single memory address space. We say two strands are logically parallel if they are not ordered in the dependence graph. Logically parallel reads (i.e., logically parallel strands reading the same memory location) are permitted, but not determinacy races (i.e., logically parallel strands that read or write the same location with at least one write).

Schedule. A schedule for a task is a "valid" mapping of its strands to processor cores across time. A scheduler is a tool that creates a valid schedule for a task (or a computation) on a machine, reacting to the behavior (execution time, etc.) of the strands on the machine. In this section, we define what constitutes a valid schedule for a nested parallel computation on a machine. We restrict ourselves to nonpreemptive schedulers-schedulers that cannot migrate strands across cores once they begin executing. Both work-stealing and space-bounded schedulers are nonpreemptive.

A nonpreemptive schedule for a task $\mathrm{t}$ defines for each strand in $\ell \in \mathrm{L}(\mathrm{t})$,

-start time: the time the first instruction of $\ell$ begins executing;

- end time: the (post facto) time the last instruction of $\ell$ finishes; and

-location: the core on which the strand $\ell$ is executed, denoted core $(\ell)$.

We say that a strand $\ell$ is live between its start and end time. Note that this duration is a function of both the strand and the machine.

A nonpreemptive schedule must also obey the following constraints:

- (ordering): Any strand $\ell_{1}$ ordered before $\ell_{2}$ in the dependence graph must end before $\ell_{2}$ starts.

-(nonpreemptive execution): No two strands may be live on the same core at the same time.

We extend some of this notation and terminology to tasks. The start time of a task $t$ is the start time of the first strand in t. Similarly, the end time of a task is the end time of the last strand in t. A task may be live on multiple cores at the same time.

When discussing specific schedulers, it is convenient to consider the time a task or strand first becomes available to execute. We use the term spawn time to refer to this time, which is the instant at which the preceding fork or join finishes. Naturally, the spawn time is no later than the start time, but a schedule may choose not to execute the task or strand immediately. We say that the task or strand is queued during the time between its spawn time and start time as it is most often put on hold in some queue, awaiting execution, by a scheduler. When a scheduler is able to reserve sufficient processing and memory resources for a task or a strand, it pulls it out of the queue and starts execution, at which point it becomes live. Figure 2 (right) illustrates the spawn, start, and end times of a task and its initial strand. The task and initial strand are spawned and start at the same time by definition. The strand 


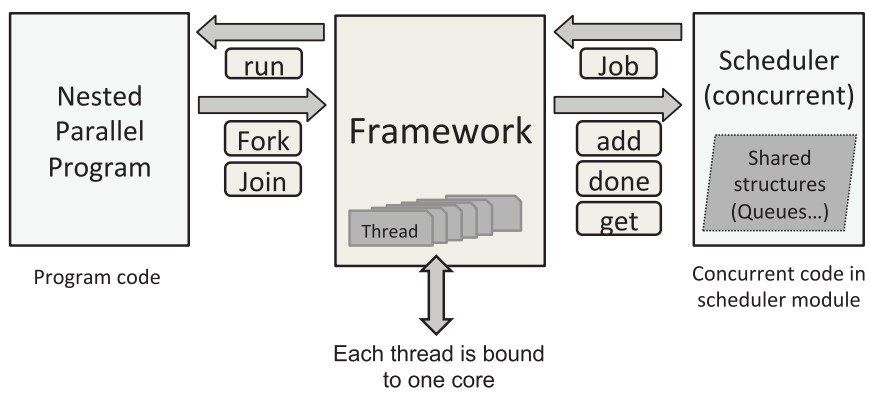

Fig. 3. Interface for the program and scheduling modules.

is continuously executed until it ends, while a task goes through several phases of execution and idling before it ends.

Machine Model: Parallel Memory Hierarchy (PMH). Following prior work addressing multilevel parallel hierarchies [Alpern et al. 1993; Chowdhury and Ramachandran 2007; Blelloch et al. 2008; Chowdhury and Ramachandran 2008; Valiant 2011; Blelloch et al. 2010; Chowdhury et al. 2010, 2013; Blelloch et al. 2011], we model parallel machines using a tree-of-caches abstraction. For concreteness, we use a symmetric variant of the PMH model [Alpern et al. 1993] (see Figure 1(b)), which is consistent with many other models [Blelloch et al. 2008, 2010; Chowdhury and Ramachandran 2007, 2008; Chowdhury et al. 2010, 2013]. A PMH consists of a height- $h$ tree of memory units, called caches. The leaves of the tree are at level-0 and any internal node has level one greater than its children. The leaves (level-0 nodes) are cores, and the level- $h$ root corresponds to an infinitely large main memory. As described in Blelloch et al. [2011], each level in the tree is parameterized by four parameters: the size of the cache $M_{i}$ at level $i$; the block size $B_{i}$ used to transfer to the next higher level; the cost of a cache miss $C_{i}$, which represents the combined costs of latency and bandwidth; and the fanout $f_{i}$ (number of level $i-1$ caches below it).

\section{EXPERIMENTAL FRAMEWORK}

We implemented a C++-based framework in which nested parallel programs and schedulers can be built for shared-memory multicore machines. The implementation, along with a few schedulers and algorithms, is available on the web page http://www.cs.cmu.edu/ hsimhadr/sched-exp. Some of the code for the threadpool module has been adapted from an earlier implementation of threadpool [Kriemann 2004]. Our framework was designed with the following objectives in mind.

Modularity: The framework separates the specification of three componentsprograms, schedulers, and description of machine parameters-for portability and fairness as depicted in Figure 3. The user can choose any of the candidates from these three categories. Note, however, that some schedulers may not be able to execute programs without scheduler-specific hints (such as space annotations).

Composable Interface: The interface for specifying the components should be composable, and the specification built on the interface should be easy to reason about.

Hint Passing: While it is important to separate program and schedulers, it is useful to allow the program to pass hints (extra annotations on tasks) to the scheduler to guide its decisions.

Minimal Overhead: The framework itself should be lightweight with minimal system calls, locking, and code complexity. The control flow should pass between 
the functional modules (program, scheduler) with negligible time spent outside. The framework should avoid generating background memory traffic and interrupts.

Timing and Measurement: It should enable fine-grained measurements of the various modules. Measurements include not only clock time but also insightful hardware counters such as cache and memory traffic statistics. In light of the preceding objective, the framework should avoid OS system calls for these, and should use direct assembly instructions.

\subsection{Interface}

The framework is a threadpool that has separate interfaces for the program and the scheduler (see Figure 3). The programming interface allows the specification of tasks to be executed and the ordering constraints between them, while the scheduler interface allows the specification of the ordering policy within the allowed constraints.

Programs: Nested parallel programs, with no other synchronization primitives, are composed from tasks using fork and join constructs, which correspond to the fork and join points in the computation model defined in Section 2 and illustrated in Figure 2 (left). A parallel_for primitive built with fork and join is also provided.

A computation is specified with classes that inherit the Job class. Each inherited class of the Job class overrides a method to specify some sequential code that always logically terminates in a fork or a join call. Further, this is the only place where fork and join calls are allowed. An instance of a class derived from the Job class is identified with a strand. Different strands nested immediately within a task correspond to distinct instances of a derived Job class. The arguments passed to a fork call are (1) a list of instances of the Job class that specifies the first strand in the subtasks that constitute the parallel block it starts, and (2) an instance of the Job class that specifies the continuation strand to be executed after the corresponding join. Thus, a task as defined in the computation model corresponds to the composition of several instances of the Job class, one for each strand in it. At times, we loosely refer to the task corresponding to an instance of Job class. This should be taken to mean the task within which the strand corresponding to the instance of the Job class is immediately nested. This interface could be readily extended to handle nonnested parallel constructs such as futures [Spoonhower et al. 2009] by adding other primitives to the interface beyond fork and join.

The interface allows extra annotations on a task such as its size, which is required by space-bounded schedulers. Such tasks inherit a derived class of Job class, the extensions in the derived class specifying the annotations. For example, the class SBJob suited for space-bounded schedulers is derived from Job by adding two functions-size (uint) and strand_size(uint) — that allow the annotations of the job size. These methods specify the size as a function of an abstract parameter called block_size. The function itself is independent of the machine. When called with the block_size set to the actual size of cache blocks on the target machine, the function returns the space used on the machine. Specifying the size as a function is particularly useful for expressing the impact of block sizes on the footprints of sparse data layouts.

Scheduler: The scheduler is a concurrent module that handles queued and live tasks (as defined in Section 2) and is responsible for maintaining its own queues and other internal shared data structures. The module interacts with the framework that consists of a thread attached to each processing core on the machine, through an interface with three call-back functions.

-Job* get (ThreadIdType): This is called by the framework on behalf of a thread attached to a core when the core is ready to execute a new strand, after completing a 


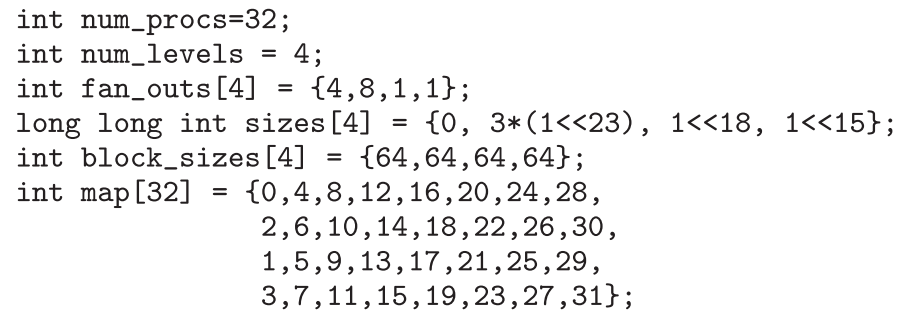

Fig. 4. Specification entry for a 32-core Xeon ${ }^{\circledR}$ machine depicted in Figure 1(a).

previously live strand. The function may change the internal state of the scheduler module and return a (possibly null) Job so that the core may immediately begin executing the strand. This function specifies core for the strand.

-void done(Job*, ThreadIdType): This is called when a core finishes the execution of a strand. The scheduler is allowed to update its internal state to reflect this completion.

— void add (Job*, ThreadIdType): This is called when a fork or join is encountered. In case of a fork, this call-back is invoked once for each of the newly spawned tasks. For a join, it is invoked for the continuation task of the join. This function decides where to enqueue the job.

Other auxiliary parameters to these call-backs have been dropped from the previous description for clarity and brevity. An argument of type Job* passed to these functions may be an instance of one of the derived classes of Job* that carries additional information helpful to the scheduler. Appendix B presents an example of a work-stealing scheduler implemented in this scheduler interface.

Machine Configuration: The interface for specifying machine descriptions accepts a description of the cache hierarchy: number of levels, fanout at each level, and cache and cache-line size at each level. In addition, a mapping between the logical numbering of cores on the system to their left-to-right position as a leaf in the tree of caches must be specified. For example, Figure 4 is a description of one Nehalem-EX series 4 -socket $\times 8$ core machine (32 physical cores) with three levels of caches as depicted in Figure 1(a). This specification of cache sizes assumes inclusive caches. The interaction between noninclusive caches and the schedulers we use is complicated. One way to use this interface to describe noninclusive caches could be to specify the size of a cache in the interface to be the sum of its size and that of all the caches below it.

\subsection{Implementation}

The runtime system initially fixes a POSIX thread to each core. Each thread then repeatedly performs a call (get) to the scheduler module to ask for work. Once assigned a task and a specific strand inside it, the thread completes the strand and asks for more work. Each strand ends in either a fork or a join. In either scenario, the framework invokes the done call-back. For a fork (join), the add call-back is invoked to let the scheduler add new tasks (the continuation task, respectively) to its data structures.

All specifics of how the scheduler operates (e.g., how the scheduler handles work requests, whether it is distributed or centralized, internal data structures, where mutual exclusion occurs, etc.) are relegated to scheduler implementations. Outside the scheduling modules, the runtime system includes no locks, synchronization, or system calls (except during the initialization and cleanup of the thread pool), meeting our design objective. 


\subsection{Measurements}

Active Time and Overheads: Control flow on each thread moves between the program and the scheduler modules. Fine-grained timers in the framework break down the execution time into five components: (1) active time- - the time spent executing the program, (2) add overhead, (3) done overhead, (4) get overhead, and (5) empty queue overhead. While active time depends on the number of instructions and the communication costs of the program, add, done, and get overheads depend on the complexity of the scheduler and the number of times the scheduler code is invoked by forks and joins. The empty queue overhead is the amount of time the scheduler fails to assign work to a thread (get returns null). Any one thread taking longer than others would keep the queues of all the other threads empty, adding to their empty queue overhead. Thus, the average empty queue time captures the load-balancing capability of the scheduler. In most of the results in Section 5, we usually report two numbers: active time averaged over all threads and the average overhead, which includes measures (2) through (5). Note that while we might expect this partition of time to be independent, it is not so in practice-the background coherence traffic generated by the scheduler's bookkeeping may adversely affect active time. The timers have very little overhead in practice-less than $1 \%$ in most of our experiments.

Measuring Hardware Counters: Modern multicores are equipped with hardware counters that can provide various performance statistics such as the number of cache misses at various levels. Such counters, however, are somewhat challenging to use. Appendix C details the specific methodology we used for the Intel ${ }^{\circledR}$ Nehalem-EX architecture.

\section{SCHEDULERS}

In this section, we will define the class of space-bounded schedulers and describe the schedulers we compare using our framework.

\subsection{Space-Bounded Schedulers}

Space-bounded schedulers are designed to achieve good cache performance on PMHs and use the sizes of tasks and strands to choose a schedule mapping the hierarchy of tasks in a nested parallel program to the hierarchy of caches. They have been described previously in Chowdhury et al. [2010] and Blelloch et al. [2011]. The definitions in Chowdhury et al. [2010] are not complete in that they do not specify how to handle "skip level" tasks (described later). Our earlier definition [Blelloch et al. 2011] handled skip-level tasks but was more restrictive than necessary, ruling out practical variants, as discussed later. Here, we provide a slightly broader definition for the class of space-bounded schedulers that allows for practical variants, while retaining the strong analytical bounds on cache misses that are the hallmark of the class of space-bounded schedulers. Specifically, our new definition provides more flexibility in the scheduling of strands by slightly relaxing the size restrictions for strands (through a new parameter $\mu$ ). We also allow strand sizes to be annotated directly rather than inheriting the size of the enclosing task.

Informally, a space-bounded schedule satisfies two properties: (1) Anchored: each task t gets "anchored" to the smallest possible cache that is larger than its size-strands within t can only be scheduled on cores in the tree rooted at the cache; and (2) Bounded: at any point in time, the sum of the sizes of all tasks and strands occupying space in a cache is at most the size of the cache. These two conditions (when formally defined) are sufficient to imply strong bounds on the number of cache misses at every level in the tree of caches. A good space-bounded scheduler would also handle load balancing subject to anchoring constraints to quickly complete execution. 
More formally, a space-bounded scheduler is parameterized by a global dilation parameter $0<\sigma \leq 1$ and machine parameters $\left\{M_{i}, B_{i}, C_{i}, f_{i}\right\}$. We will need the following terminologies for the definition (which are both simplified and generalized from Blelloch et al. [2011]).

Task Size and Strand Size: The size of a task (strand) is defined as a function of cacheline size $B$, independent of the scheduler. Let $l o c(t ; B)$ denote the set of distinct cache lines touched by instructions within a task $\mathrm{t}$. Then $S(\mathrm{t} ; B)=|\operatorname{loc}(\mathrm{t} ; B)| \cdot B$ denotes the size of t. The size of a strand is defined in the same way. While results in Blelloch et al. [2011] show that it is not necessary for the analytical bounds that strands be allowed to have their own size, and indeed strands with different sizes could be wrapped in tasks, where applicable this flexibility reduces runtime overhead. ${ }^{1}$ Note that even strands immediately nested within the same task may have different sizes.

Cluster: For any cache $X_{i}$, its cluster is the set of caches and cores nested below $X_{i}$. Let $P\left(X_{i}\right)$ denote the set of cores in $X_{i}$ 's cluster.

Befitting Cache: Given a particular cache hierarchy and dilation parameter $\sigma \in(0,1]$, we say that a level- $i$ cache befits a task $\mathrm{t}$ if $\sigma M_{i-1}<S\left(\mathrm{t}, B_{i}\right) \leq \sigma M_{i}$. The dilation parameter determines which tasks are "maximal."

Maximal Task: We say that a task $t$ with parent task $t^{\prime}$ is level-i maximal if and only if a level- $i$ cache befits $\mathrm{t}$ but not $\mathrm{t}^{\prime}$, that is, $\sigma M_{i-1}<S\left(\mathrm{t}, B_{i}\right) \leq \sigma M_{i}<S\left(\mathrm{t}^{\prime}, B_{i}\right)$. Although it might seem natural to set $\sigma=1$, we will see that setting it to a lower value like 0.5 allows multiple tasks to be anchored to a cache, enabling greater load-balancing flexibility (at a cost to locality).

Anchored: A task $\mathrm{t}$ with strand set $\mathrm{L}(t)$ is said to be anchored to level- $i$ cache $X_{i}$ (or equivalently to $X_{i}$ 's cluster) if and only if (1) it is executed entirely in the cluster, that is, $\{\operatorname{core}(\ell) \mid \ell \in \mathrm{L}(t)\} \subseteq P\left(X_{i}\right)$, and (2) the cache befits the task. Anchoring prevents the migration of tasks to a different cluster or cache. The advantage of anchoring a task to a befitting cache is that once it loads its working set, it can reuse the working set without the risk of losing it from the cache. If a task is not anchored anywhere, for notational convenience we assume it is anchored at the root of the tree.

Cache-Occupying Tasks: For a level- $i$ cache $X_{i}$ and time $\tau$, the set of cacheoccupying tasks, denoted by $\operatorname{Ot}\left(X_{i}, \tau\right)$, are the tasks that consume space in the cache at time $\tau$. The precise definition depends on whether the cache is inclusive or noninclusive. For an inclusive cache, $\operatorname{Ot}\left(X_{i}, \tau\right)$ is the union of (1) the maximal tasks live at time $\tau$ that are anchored to $X_{i}$ and (2) the maximal tasks live at time $\tau$ that are anchored to any cache below $X_{i}$ in the hierarchy whose immediate parents are anchored to a cache above $X_{i}$ in the hierarchy. The tasks in (2) are called "skip level" tasks. For a noninclusive cache, only type (1) tasks are included in $\operatorname{Ot}\left(X_{i}, \tau\right)$. Note that we need account only for maximal tasks because any nonmaximal task $t^{\prime}$ is anchored to the same cache as its closest enclosing maximal task $\mathrm{t}$ and $\operatorname{loc}\left(\mathrm{t}^{\prime} ; B\right) \subseteq \operatorname{loc}(\mathrm{t} ; B)$.

Cache-Occupying Strands: The set of cache-occupying strands for a cache $X_{i}$ at time $\tau$, denoted by $\mathrm{Ol}\left(X_{i}, \tau\right)$, is the set of strands $\{\ell\}$ such that (1) $\ell$ is live at time $\tau$; (2) $\ell$ is executed below $X_{i}$, that is, $\operatorname{core}(\ell) \in P\left(X_{i}\right)$; and (3) $\ell$ 's task is anchored strictly above $X_{i}$.

A space-bounded scheduler for a particular cache hierarchy is a scheduler parameterized by $\sigma \in(0,1]$ that satisfies the following two properties:

\footnotetext{
${ }^{1}$ On the other hand, it does require additional size information on programs-thus, we view it as optional: any strand whose size is not specified is assumed by default to be the size of its enclosing task.
} 
-Anchored: Every subtask (recursively) of the root task is anchored to a befitting cache.

-Bounded: At every time $\tau$, for every level- $i$ cache $X_{i}$, the sum of the sizes of cacheoccupying tasks and strands is at most $M_{i}$ :

$$
\Sigma_{\mathrm{t} \in \mathrm{Ot}\left(X_{i}, \tau\right)} S\left(\mathrm{t}, B_{i}\right)+\Sigma_{\ell \in \mathrm{Ol}\left(X_{i}, \tau\right)} S\left(\ell, B_{i}\right) \leq M_{i} .
$$

A key property of the definition of space-bounded schedulers in Blelloch et al. [2011] is that for any PMH and any level $i$, one can upper bound the number of level- $i$ cache misses incurred by executing a task on the PMH by $Q^{*}\left(\mathrm{t} ; \sigma M_{i}, B_{i}\right)$, where $Q^{*}$ is the parallel cache complexity as defined in the Parallel Cache Complexity (PCC) framework in Simhadri [2013]. Roughly speaking, the parallel cache complexity $Q^{*}$ of a task $t$ in terms of a cache of size $M$ and line size $B$ is defined as follows. Decompose the task into a collection of maximal subtasks that fit in $M$ space, and "glue nodes"instructions outside these subtasks. For a maximal size $M$ task $\mathrm{t}^{\prime}$, the parallel cache complexity $Q^{*}\left(\mathrm{t}^{\prime} ; M ; B\right)$ is defined to be the number of distinct cache lines it accesses. The model then pessimistically counts all memory instructions that fall outside a maximal subtask (i.e., glue nodes) as cache misses and counts accesses to a cache line from logically parallel maximal tasks multiple times. The total cache complexity of an algorithm is the sum of the complexities of the maximal subtasks and the memory accesses outside of maximal subtasks. Note that $Q^{*}$ is a program-centric or machineindependent metric, capturing the inherent locality in a parallel algorithm [Blelloch et al. 2013; Simhadri 2013].

THEOREM 4.1. Consider a PMH and any dilation parameter $0<\sigma \leq 1$. Let $\mathrm{t}$ be a task anchored to the root of the tree. Then the number of level-i cache misses incurred by executing $\mathrm{t}$ with any space-bounded scheduler is at most $Q^{*}\left(\mathrm{t} ; \sigma M_{i}, B_{i}\right)$, where $Q^{*}$ is the parallel cache complexity as defined in the PCC framework in Simhadri [2013].

The proof is a simple adaptation of the proof in Blelloch et al. [2011] to account for our more general definition. At a high level, the argument is that for any cache $X_{i}$, the cache-occupying tasks and strands for $X_{i}$ bring their working sets into the cache $X_{i}$ exactly once because the boundedness property prevents cache overflows. Thus, a replacement policy that keeps these working sets in the cache until they are no longer needed will incur no additional misses; because the PMH model assumes an ideal replacement policy, it will perform at least as well.

Note that while setting $\sigma$ to 1 yields the best bounds on cache misses, it also makes load balancing harder. As we will see later, a lower value for $\sigma$ like 0.5 enables greater scheduling flexibility.

In the course of our experimental study, we found that the following minor modification to the boundedness property of space-bounded schedulers improves their performance. Namely, we introduce a new parameter $\mu \in(0,1](\mu=0.2$ in our experiments) and modify the boundedness property to be such that at every time $\tau$, for every level- $i$ cache $X_{i}$ :

$$
\Sigma_{\mathrm{t} \in \mathrm{Ot}\left(X_{i}, \tau\right)} S\left(\mathrm{t}, B_{i}\right)+\Sigma_{\ell \in \mathrm{Ol}\left(X_{i}, \tau\right)} \min \left\{\mu M_{i}, S\left(\ell, B_{i}\right)\right\} \leq M_{i}
$$

The minimum term with $\mu M_{i}$ is to enable several large strands to be explored simultaneously without their space measure taking too much of the space bound. To understand the problem with Equation (1) (i.e., no $\mu$ parameter), consider a typical divide-and-conquer computation. The highest level of the recursion corresponds to a very large task, with potentially large strands preceding each parallel recursion. Using Equation (1), parallel scheduling is only allowed once the recursion gets far enough so that the subproblems fit in cache, which greatly limits the parallelism at the beginning of the computation. With Equation (2) and $\mu \ll 1$, the parallelism is exposed sooner 
(albeit at a cost to cache performance). This helps the scheduler to quickly traverse the higher levels of recursion in the computation and reveal parallelism, so that the scheduler can achieve better load balance.

Given this modified boundedness condition, it is easy to show that the bound in Theorem 4.1 becomes $Q^{*}\left(\mathrm{t} ; \mu \sigma M_{i}, B_{i}\right)$.

In addition to introducing space-bounded schedulers, Chowdhury et al. [2010] propose two other approaches to scheduling: CGC (coarse-grained contiguous) and CGCon-SB (which combines CGC with space-bounded schedulers). CGC schedules parallel loops and is designed to keep nearby blocks of iterations close together so they are run on the same cache. This can be simulated in our framework by grouping iterations recursively as a tree, which is what we do. This means that for us, a loop of length $n$ will require at least $\log n$ depth, while CGC technically allows a loop in constant depth. For the modest number of cores we experiment with, this will not have much effect, but perhaps for a significantly larger number of cores it would be worth considering directly scheduling loops as in CGC. CGC-on-SB is primarily a mechanism for skipping levels in the cache. Our variant of a space-bounded scheduler already allows for level skipping.

\subsection{Schedulers Implemented}

Space-Bounded Schedulers: SB and SB-D. We implemented a space-bounded scheduler by constructing a tree of caches based on the specification of the target machine. Each cache is assigned one logical queue, a counter to keep track of "occupied" space, and a lock to protect updates to the counter and queue. Cores can be considered to be leaves of the tree; when a scheduler call-back is issued to a thread, that thread can modify an internal node of the tree after gathering all locks on the path to the node from the core it is mapped onto. This scheduler accepts Jobs that are annotated with task and strand sizes. When a new Job is spawned at a fork, the add call-back enqueues it at the cluster where its parent was anchored. For a new Job spawned at a join, add enqueues it at the cluster where the Job that called the corresponding fork of this join was anchored.

A basic version of such a scheduler would implement logical queues at each cache as one queue. However, this presents two problems: (1) it is difficult to separate tasks in queues by the level of cache that befits it, and (2) a single queue might be a contention hotspot. To solve problem (1), behind each logical queue, we use separate "buckets" for each level of cache below to hold tasks that befit those levels. Cores looking for a task at a cache go through these buckets from the top (bucket corresponding to the cache immediately below) to the bottom (bucket corresponding to the smallest cache in the hierarchy). We refer to this variant as the SB scheduler. To solve problem (2) involving queueing hotspots, we replace the top bucket with a distributed queue-one queue for each child cache-like in the work-stealing scheduler. We refer to the SB scheduler with this modification as the SB-D scheduler.

Work-Stealing Scheduler: WS. A basic work-stealing scheduler based on Cilk++ [Blumofe and Leiserson 1999] is implemented and is referred to as the WS scheduler. Since the Cilk++ runtime system is built around work stealing and deeply integrated and optimized exclusively for it, we focus our comparison on the WS implementation in our framework for fairness and to allow us to implement variants. The head-tohead microbenchmark study in Section 5 between our WS implementation and Cilk ${ }^{\mathrm{TM}}$ Plus (the commercial version of Cilk++) suggests that, for these benchmarks, WS well represents the performance of Cilk++'s work stealing.

We associate a double-ended queue (dequeue) of ready tasks with each core. The function add enqueues new tasks (actually the first strand in the task) spawned on a 
core to the bottom of its dequeue. When in need of work, the core uses get to remove a task from the bottom of its dequeue. If its dequeue is empty, it chooses another dequeue uniformly at random and steals the work by removing a task from the top of that core's dequeue. In contrast with Cilk++, where the core that executes the last task to join carries on with the continuation strand, our implementation of WS enqueues the continuation strand at the bottom of the queue of the core that executes the last task to join. While it is possible for the same core to immediately dequeue and proceed with the continuation, the separate enqueue and dequeue calls occasionally allow other cores to steal the continuation from the queue. However, this does not significantly affect the number of cache misses in relation to the estimate given by the parallel cache complexity metric, as this metric does not count cache reuse between a parallel block and its continuation.

The only contention in this type of scheduler is on the distributed dequeues-there is no other centralized data structure. To implement the dequeues, we employed a simple two-locks-per-dequeue approach, one associated with the owning core, and the second associated with all cores currently attempting to steal. Remote cores need to obtain the second lock before they attempt to lock the first. Contention is thus minimized for the common case where the core needs to obtain only the first lock before it asks for work from its own dequeue.

Priority Work-Stealing Scheduler: PWS. Unlike in the basic WS scheduler, cores in the PWS scheduler [Quintin and Wagner 2010] choose victims of their steals according to the "closeness" of the victims in the socket layout. Dequeues at cores that are closer in the cache hierarchy are chosen with a higher probability than those that are farther away, in order to improve scheduling locality. On the other hand, the increase in probability is bounded in order to retain the good load-balancing properties of work-stealing schedulers.

\section{EXPERIMENTS}

The goal of our experimental study is to compare the performance of the four schedulers on a range of benchmarks, varying the available memory bandwidth. Our primary metrics are runtime and L3 (last-level) cache misses. We have found that the cache misses on other levels do not vary significantly among the schedulers (within 5\%). We will also validate our work-stealing implementation via a comparison with the commercial Cilk ${ }^{\mathrm{TM}}$ Plus scheduler. Next, we will quantify the overheads for spacebounded schedulers, comparing them to the overheads for work-stealing schedulers. Finally, we will study the performance impact of the key parameters $\mu$ and $\sigma$ for space-bounded schedulers.

\subsection{Benchmarks}

We use 10 benchmarks in our study. The first two are synthetic microbenchmarks that mimic the behavior of memory-intensive divide-and-conquer algorithms. Because of their simplicity, we use these benchmarks to closely analyze the behavior of the schedulers under various conditions and verify that we get the expected cache behavior on a real machine. The remaining eight benchmarks are a set of popular algorithmic kernels.

Recursive Repeated Map (RRM): This benchmark takes two $n$-length arrays $A$ and $B$ and a pointwise map function that maps elements of $A$ to $B$. In our experiments, each element of the arrays is a double and the function simply adds one. RRM first does a parallel pointwise map from $A$ to $B$ and repeats the same operation multiple times. It then divides $A$ and $B$ into two by some ratio (e.g., 50/50) and recursively calls the same operation on each of the two parts. The base case of the recursion is set to 
some constant $(1<<13$ in our experiments) at which point the recursion terminates. The input parameters are the size of the arrays $n$, number of repeats $r$, the cut ratio $f$, and the base-case size. We set $r=3$ (to imitate the number of times the input array is touched in our quicksort) and $f=50 \%$ in the experiments unless otherwise noted. RRM is a memory-intensive benchmark because there is very little work done per memory operation. However, once a recursive call fits in a cache (i.e., the cache size is at least $16 n$ bytes for subproblem size $n$ ), all remaining accesses are cache hits.

Recursive Repeated Gather (RRG): This benchmark is similar to RRM, but instead of doing a simple map, it does a gather. In particular, at any given level of the recursion, it takes three $n$-length arrays $A, B$, and $I$ and for each location $i$ sets $B[i]=A[I[i]$ $\bmod n$ ]. The values in $I$ are random integers. As with RRM, after repeating $r$ times, it splits the arrays in two and repeats on each part. RRG is even more memory intensive than RRM because its accesses are random instead of linear. Again, however, once a recursive call fits in a cache, all remaining accesses are cache hits.

Quicksort: This is a parallel quicksort algorithm that parallelizes both the partitioning and the recursive calls, using a median-of-3 pivot selection strategy. It switches to a version that parallelizes only the recursive calls for $n<128 \mathrm{~K}$ and a serial version for $n<16 K$. These parameters worked well for all the schedulers. We note that our quicksort is about $2 \times$ faster than the Cilk code found in the Cilk+ guide [Leiserson 2010]. This is because it does the partitioning in parallel. It is also the case that the divide does not exactly partition the data evenly, because it depends on how well the pivot divides the data. For an input of size $n$, the program has cache complexity $Q^{*}(n ; M, B)=O\left(\lceil n / B\rceil \log _{2}(n / M)\right)$ and therefore is reasonably memory intensive.

Samplesort: This is the cache-optimal parallel Sample Sort algorithm described in Blelloch et al. [2010]. The algorithm splits the input of size $n$ into $\sqrt{n}$ subarrays, recursively sorts each subarray, "block transposes" them into $\sqrt{n}$ buckets, and recursively sorts these buckets. For this algorithm, $Q^{*}(n ; M, B)=O\left(\lceil n / B\rceil \log _{2+(M / B)} n / B\right)$, making it relatively cache friendly and optimally cache oblivious even.

Aware Samplesort: This is a variant of sample-sort that is aware of the cache sizes. In particular, it moves elements into buckets that fit into the L3 cache and then runs quicksort on the buckets. This is the fastest sort we implemented and is in fact faster than any other sort we found for this machine. In particular, it is about $10 \%$ faster than the PBBS sample sort [Shun et al. 2012].

Quad-Tree: This generates a quad tree for a set of $n$ points in two dimensions. This is implemented by recursively partitioning the points into four sets along the midline of each of two dimensions. When the number of points is less than $16 \mathrm{~K}$, we revert to a sequential algorithm.

Quickhull: This is a recursive two-way divide-and-conquer algorithm for finding the convex hull of points on a 2-dimensional plane. The recursion is very similar to quicksort. We switch to a serial version when the number of points is less than $16 \mathrm{~K}$. Although the performance of this deterministic algorithm is bad for worst-case inputs, it does reasonably well on most inputs. We use a set of $n$ uniformly random points on a 2-sphere as our input. For this input, the program has cache complexity $Q^{*}(n ; M, B)=O\left(\lceil n / B\rceil \log _{2}(n / M)\right)$ and therefore is reasonably memory intensive.

Matrix Multiplication: This benchmark is an eight-way recursive matrix multiplication. To allow for an in-place implementation, four of the recursive calls are invoked in parallel, followed by the other four. Matrix multiplication has cache complexity $Q^{*}(n ; M, B)=\left(\left\lceil n^{2} / B\right\rceil \times\lceil n / \sqrt{M}\rceil\right)$. The ratio of instructions to cache misses is therefore 
very high, about $B \sqrt{M}$, making this a very compute-intensive benchmark. We switch to serial Intel ${ }^{\circledR}$ Math Kernel Library's cblas_dgemm matrix multiplication for sizes of $\leq 128 \times 128$ to make use of the floating-point SIMD operations.

Triangular Solver: This benchmark solves the equation $U X=B$ for $X$, where $U$ is a dense upper triangular matrix of order $n$ and $B$ is an $n \times m$ matrix. The algorithm is recursive. It first partitions $U$ into two upper triangular matrices $U_{1}, U_{2}$ of order $n / 2$ and a square matrix $U^{\prime}$ of size $n / 2 \times n / 2$, and $B$ into four matrices $B_{11}, B_{12}, B_{21}, B_{22}$ of size $n / 2 \times m / 2$ each. Then it solves for $U_{2} X_{21}=B_{21}$ and $U_{2} X_{22}=B_{22}$ in parallel, uses the value of $X_{21}$ and $X_{22}$ along with $U^{\prime}$ to update $B_{11}, B_{12}$ using matrix multiplication, and then solves for $U_{1} X_{11}=B_{11}$ and $U_{1} X_{12}=B_{12}$ in parallel. The asymptotic cache complexity is the same as that for matrix multiplication, and its depth is $O(n \log n)$. While the natural DAG for this computation has depth $O(n)$, expressing it as a strict fork-join program introduced false dependencies and increases its depth. Also, just as in the case of matrix multiplication, we switch to Intel ${ }^{\circledR}$ Math Kernel Library's LAPACKE_dtrtrs routine for leaf nodes of size less than $128 \times 128$.

Cholesky Factorization: This benchmark factorizes a dense symmetric positive definite matrix $A$ into the form $L L^{T}$, where $L$ is a lower triangular matrix. Since $A$ is symmetric, only one of the upper or lower triangular parts is required as input. The algorithm is recursive. It partitions upper triangular matrix $A$ of order $n$ into two upper triangular matrixes $A_{1}, A_{2}$ of order $n / 2$ and a square matrix $A^{\prime}$ of size $n / 2 \times n / 2$. Recursively, it factorizes $A_{1}$, uses the factor to do a triangular solve on $A^{\prime}$ followed by a matrix multiplication on to $A_{2}$, and then factorizes $A_{2}$. The asymptotic cache complexity of the algorithm is the same as that of matrix multiplication, and the depth of the algorithm is $O\left(n \log ^{2} n\right)$. As in the previous case, false dependencies introduced by using a fork-join model increase the depth by a factor of $O\left(\log ^{2} n\right)$. The leaf nodes of the recursion use the LAPACKe_dpotrf factorization routine.

\subsection{Experimental Setup}

We ran the benchmarks on a four-socket 32-core Xeon ${ }^{\circledR} 7560$ machine (Nehalem-EX architecture), as described in Figures 1(a) and 4, using each of the four schedulers. Each core uses two-way hyperthreading. The last-level cache on each socket is a $24 \mathrm{MB}$ L3 cache that is shared by eight cores. Each of the four sockets on the machine has memory links to distinct DRAM modules. The sockets are connected with the Intel ${ }^{\circledR}$ QPI interconnect. Memory requests from a socket to a DRAM module connected to another socket pass through the QPI, the remote socket, and the remote memory link. To prevent excessive TLB cache misses, we use Linux hugepages of size $2 \mathrm{MB}$ to preallocate the space required by the algorithms. We set the value of vm.nr_hugepages to 10,000 using sysctl to create a pool of huge pages. We use the hugectl tool [Litke et al. 2006] to execute memory allocations with hugepages.

Monitoring L3 Cache. We focus on L3 cache misses, the most expensive cache level before DRAM on our machine. While we report runtime subdivided into application code and scheduler code, such partitioning was not possible for L3 misses because of software limitations. Even if it were possible to count them separately, it would be difficult to interpret the results because of the nontrivial interference between the data cached by the program and by the scheduler. Further details can be found in Appendix C.

Controlling Bandwidth. As part of our study, we quantify runtime improvements varying the available memory bandwidth per core (the "bandwidth gap"). This is motivated by trends toward increasing the number of cores per socket more rapidly than the available memory bandwidth, thereby increasing the bandwidth gap. We control 
the memory bandwidth available to the program as follows. Because the QPI has high bandwidth, if we treat the RAM as a single functional module, the bandwidth between the L3 and the RAM depends on the number of memory links used, which in turn depends on the mapping of pages to the DRAM modules. If all the pages used by a program are mapped to DRAM modules connected to one socket, the program effectively utilizes one-fourth of the memory bandwidth. On the other hand, an even distribution of pages to DRAM modules across the sockets provides the full bandwidth to the program. One way to map a page to a DRAM module associated with a specific socket is to write to a freshly allocated page from a core in the socket. The numactl tool [Kleen 2004] can be also used to control this mapping. When memory is mapped to the modules on only one socket, the latency of a memory access for the other three sockets increases (by about a factor of two [Molka et al. 2009]). We disregard this nonuniformity since the workloads are primarily constrained by bandwidth as opposed to latency. Further, the actual bandwidth available depends on many factors involved in the configuration of the hardware [Fujitsu Technology Solutions 2010] and the one-fourth bandwidth estimate for this configuration is a rough estimate.

Code. We use the same code (i.e., we do not change the task specification) for the applications/algorithms across the schedules for all benchmarks, except for the Cilk Plus code. One program for each benchmark specified through the fork-join interface is passed to all the schedulers. The code for the Cilk Plus interface is kept as close to the code in our programming interface as possible. The code always includes the space annotations, but those annotations are ignored by the schedulers that do not need them. The code was compiled with a Cilk Plus fork of gcc 4.8 .0 compiler.

\subsection{Results}

We use $\sigma=0.5$ and $\mu=0.2$ in the SB and SB-D schedulers, after some experimentation with the parameters, unless otherwise noted. In the PWS scheduler, we set the probability of an intrasocket steal to be 10 times that of an intersocket steal. All numbers reported in this article are the average of at least 10 runs with the smallest and largest readings across runs removed. The largest reading is removed to eliminate the possibility of a background process (e.g., daemon) interfering with the timing. The smallest reading is removed to compensate for this. The results are not sensitive to this particular choice of reporting. Although all the runs are short (on the order of 1 second), the duration of the runs is highly reproducible, with standard deviations under $5 \%$ for all experiments (based on 25 runs) and around 1\% to $2 \%$ for most experiments.

Synthetic Benchmarks. Figures 5 and 6 show the number of L3 cache misses of RRM and RRG, respectively, along with their active times and scheduling overheads on 64 hyperthreads at different bandwidth values. In addition to the four schedulers discussed in Section 4.2 (WS, PWS, SB , and SB-D), we include the Cilk Plus workstealing scheduler in these plots to validate our WS implementation. We could not separate overhead from time in Cilk Plus because it does not supply such information.

These plots show that the space-bounded schedulers incur roughly $42 \%$ to $44 \%$ fewer L3 cache misses than the work-stealing schedulers. As expected, the number of L3 misses does not significantly depend on the available bandwidth. On the other hand, the active time is most influenced by the number of instructions in the benchmark (constant across schedulers) and the costs incurred by L3 misses. The extent to which improvements in L3 misses translate to an improvement in active time depends on the memory bandwidth given to the program. When the bandwidth is low (25\%), the active times are almost directly proportional to the number of L3 cache misses, while at full bandwidth, the active times are far less sensitive to misses.

The difference in L3 cache costs of space-bounded and work-stealing schedulers roughly corresponds to the difference between the cache complexity of the program 


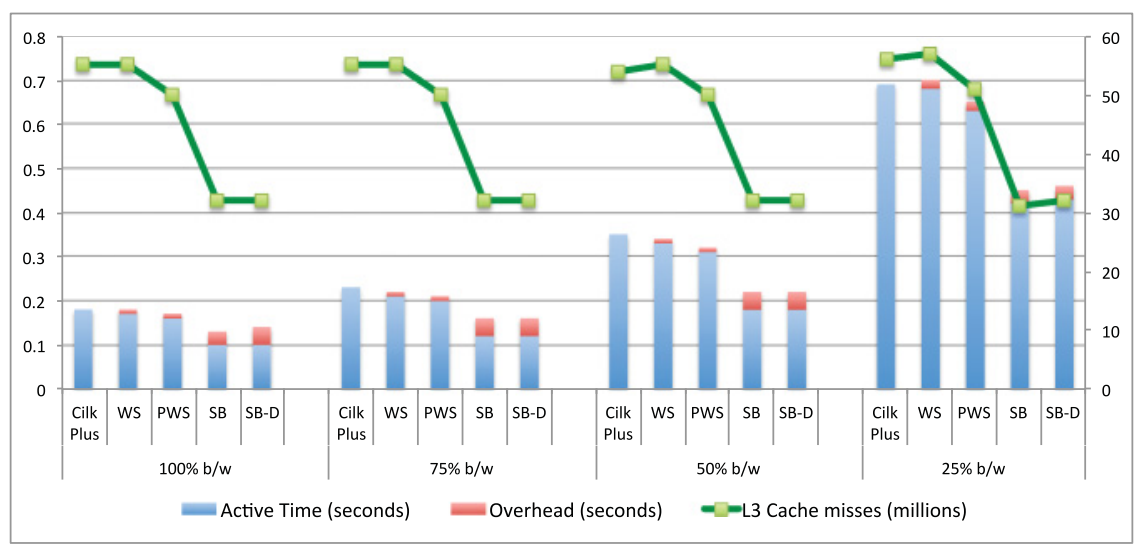

Fig. 5. RRM on 10 million double elements, varying the memory bandwidth. Left axis is running time in seconds. Right axis is L3 misses in millions.

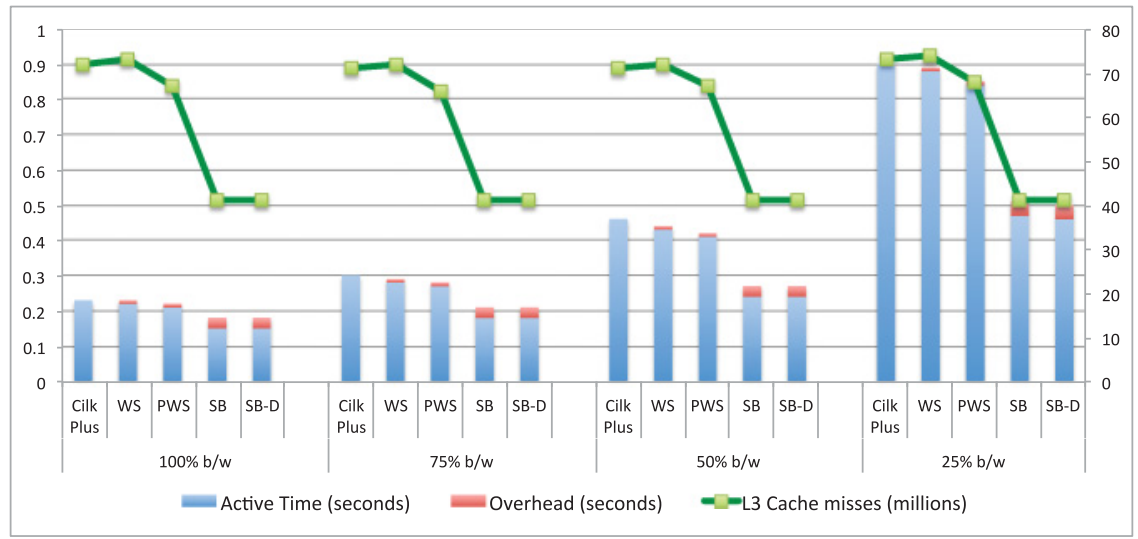

Fig. 6. RRG on 10 million double elements, varying the memory bandwidth. Left axis is running time in seconds. Right axis is L3 misses in millions.

with a cache of size $\sigma M_{3}\left(M_{3}\right.$ being the size of L3) and a cache of size $M_{3} / 16$ (because eight cores with 16 hyperthreads share an L3 cache). In other words, space-bounded schedulers share the cache constructively while work-stealing schedulers effectively split the cache between the cores. To see this, consider our RRM benchmark. Each Map operation that does not fit in L3 touches $2 \times 10^{7} \times 8=160$ million bytes of data, and RRM has to unfold four levels of recursion before it fits in $\sigma M_{3}=0.5 \times 24 \mathrm{MB}=12 \mathrm{MB}$ space with space-bounded schedulers. Therefore, because the cache line size is $B_{3}=64$ bytes, space-bounded schedulers incur about $\left(160 \times 10^{6} \times 3 \times 4\right) / 64=30 \times 10^{6}$ cache misses, which matches closely with the results in Figure 5. On the other hand, the number of cache misses of the WS scheduler (55 million) corresponds to unfolding about seven levels of recursions, three more than with space-bounded schedulers. Loosely speaking, this means that the recursion has to unravel to one-sixteenth the size of L3 before workstealing schedulers start preserving locality.

To support this observation, we ran the RRM and RRG benchmarks varying the number of cores per socket; the results are in Figure 7. The number of L3 cache misses when using SB and SB-D schedulers does not change with the number of cores, because cores constructively share the L3 cache independent of their number. However, when 


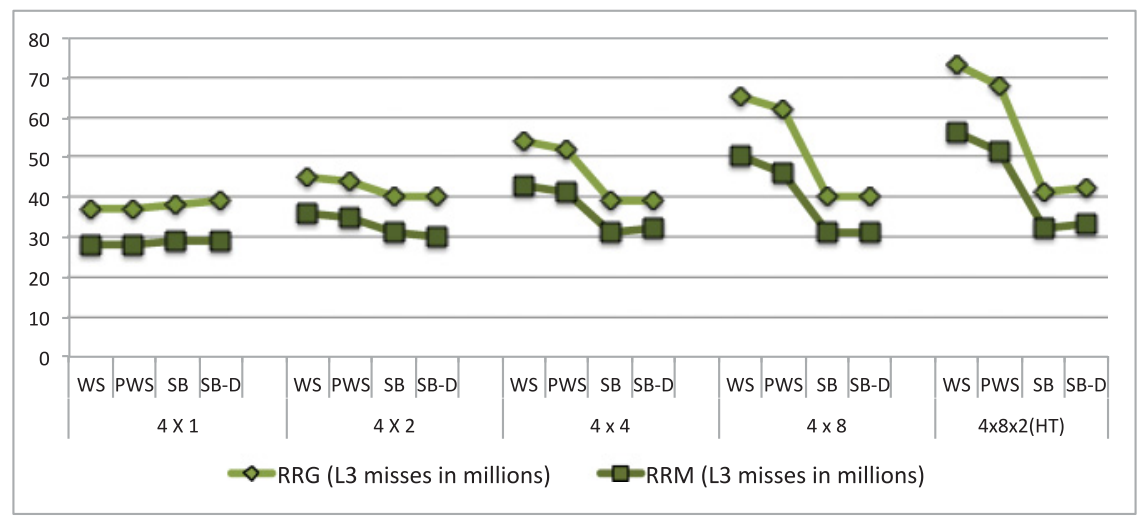

Fig. 7. L3 cache misses for RRM and RRG, varying the number of cores used per socket.

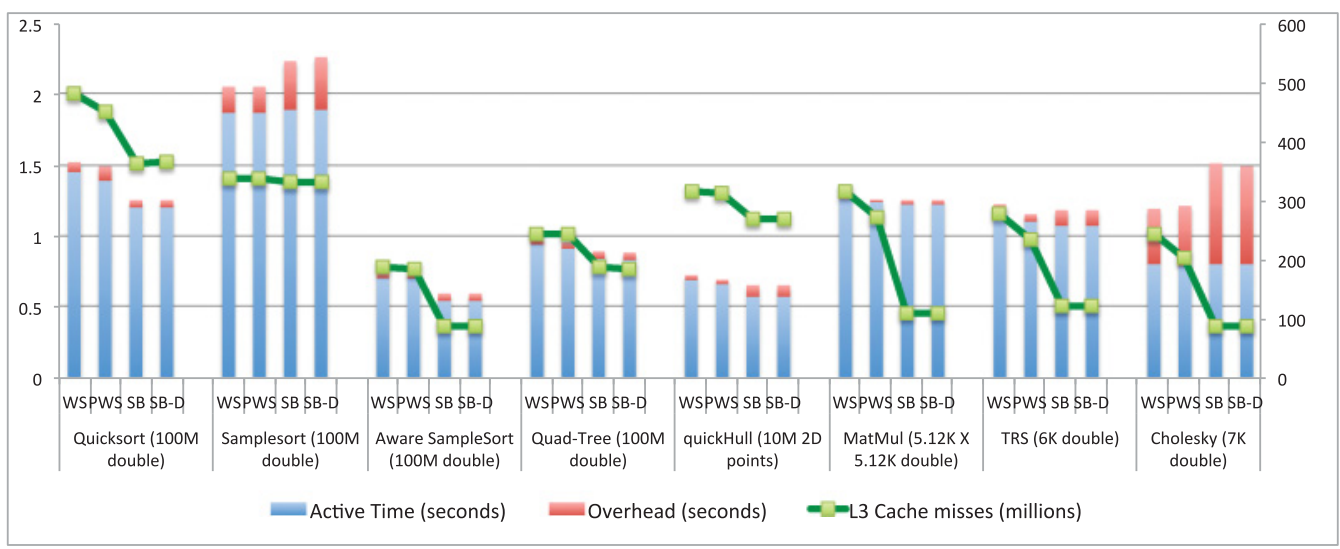

Fig. 8. Active times, overheads, and L3 cache misses for the eight benchmark algorithms at full bandwidth.

using WS and PWS schedulers, the number of L3 misses is highly dependent on the number of cores: when fewer cores are active on each socket, there is lesser contention for space in the shared L3 cache. Thus, again, the experimental results coincide with the theoretical analysis.

These experiments indicate that the advantages of space-bounded schedulers over work-stealing schedulers improve as (1) the number of cores per socket goes up, and (2) the bandwidth per core goes down. At eight cores, there is a 30\% to 35\% reduction in L3 cache misses. At 64 cores, we would expect (by the analysis) over a $60 \%$ reduction.

Algorithms. Figures 8 and 9 show the active times, scheduling overheads, and L3 cache misses of the eight algorithmic kernels at $100 \%$ and $25 \%$ bandwidth, respectively, with 64 hyperthreads. These plots show that the space-bounded schedulers incur significantly fewer L3 cache misses on seven of the eight benchmarks, with up to $65 \%$ on matrix multiply. The cache-oblivious sample sort is the sole benchmark with little difference in L3 cache misses across schedulers. Given the problem size $n=10^{8}$ doubles and sample sort's $\sqrt{n}$-way recursion, all subtasks after one level of recursion are much smaller than the L3 cache. Thus, all four schedulers avoid overflowing the cache. Because of their added overhead relative to work stealing, the space-bounded schedulers are $7 \%$ slower for this benchmark. 


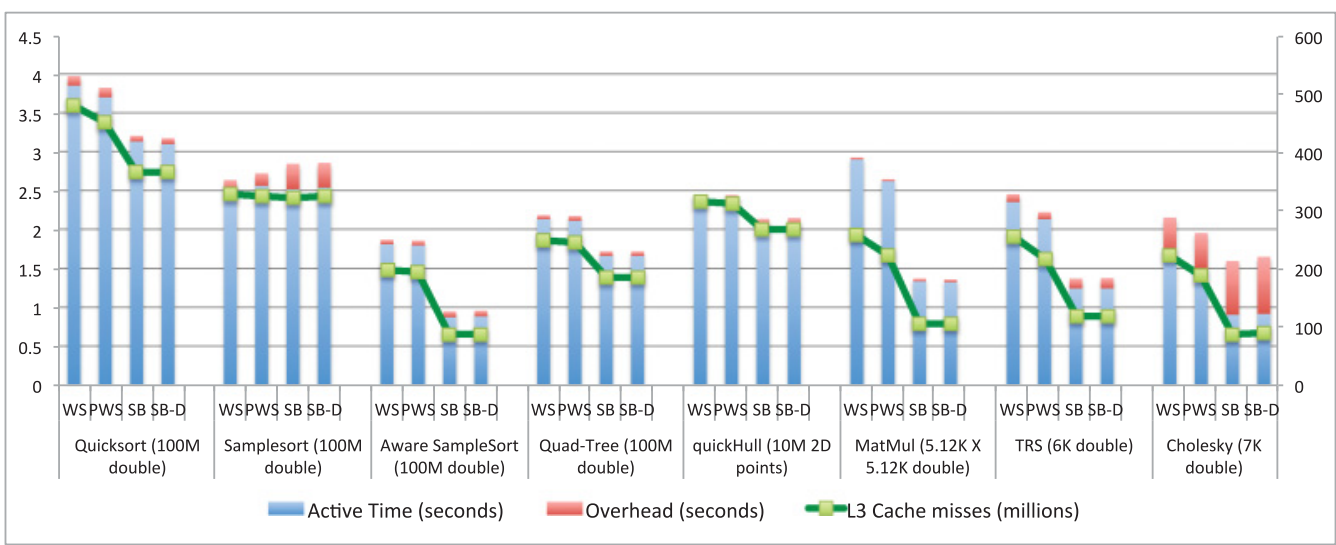

Fig. 9. Active times, overheads, and L3 cache misses for the eight benchmark algorithms at $25 \%$ bandwidth.

As with the synthetic benchmarks, the sensitivity of active time to L3 cache misses depends on whether the algorithm is memory intensive enough to stress the bandwidth. Matrix Multiplication, although benefitting from space-bounded schedulers in terms of cache misses, shows no significant improvement in active time at full bandwidth because it is very compute intensive. However, when the machine bandwidth is reduced to 25\%, Matrix Multiplication is bandwidth bound and the space-bounded schedulers are about 50\% faster than work stealing. The sorting-based benchmarksQuicksort, Aware Samplesort, Quickhull, and Quad-tree-are memory intensive and see improvements of up to $25 \%$ in running time at full bandwidth (Figure 8). At $25 \%$ bandwidth, the improvement is even more significant and up to $40 \%$ (Figure 9).

The behavior of Triangular solver and Cholesky factorization is very similar to that of Matrix Multiplication in terms of active time-insensitive to improvement in cache misses at full bandwidth and almost proportional to L3 cache misses at $25 \%$ bandwidth. This is to be expected as their work and cache complexities are very similar. However, Cholesky factorization has significantly higher overhead (27\% in the worst case), most of which is due to empty queue time- $380 \mathrm{~ms}$ of $390 \mathrm{~ms}$ for the WS scheduler and $790 \mathrm{~ms}$ of $800 \mathrm{~ms}$ for the SB scheduler at full bandwidth. This is because the recursive Cholesky factorization is serial at $O\left(n \log ^{2} n / L\right)$ points along the critical path in its DAG, where $L$ is the order of the base case of the recursion. At these points, all cores but one are idle with any scheduler. The empty queue time for the SB scheduler is significantly larger because it anchors each task based on its size, and the size of the tasks near these effectively serial points in the DAG is small. Smaller tasks are anchored to smaller caches with fewer cores, leaving other cores idle. The WS scheduler is not constrained by the anchoring property and can use more cores around these serial points. This overhead may be reduced to some extent by falsely reporting a greater size for Cholesky tasks at lower levels of recursion to force the SB scheduler to be more aggressive at load balancing. Further, Cholesky factorization could also achieve better performance through a relaxation of false dependencies introduced by expressing the algorithm in the fork-join paradigm using techniques recently introduced by Tang et al. [2015]. This would reduce the depth of the algorithm to $O(n / L)$ and remove all serial points in the DAG except the start and the end.

Overheads and the Strand Boundedness Parameter $\mu$. As noted earlier, our definition of space-bounded schedulers slightly relaxes the size restrictions for strands through a new parameter $\mu$ (Equation (2) vs. Equation (1)). Figure 10 shows the SB and SB-D 


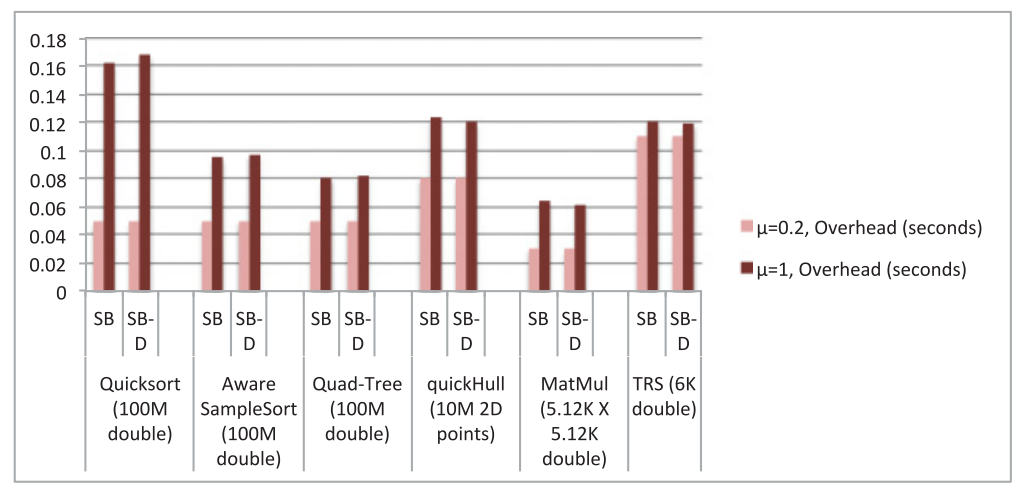

Fig. 10. Overhead of the algorithms for $\mu=0.2$ and 1.0 at full bandwidth. The overheads of Samplesort and Cholesky are much higher and not shown here.

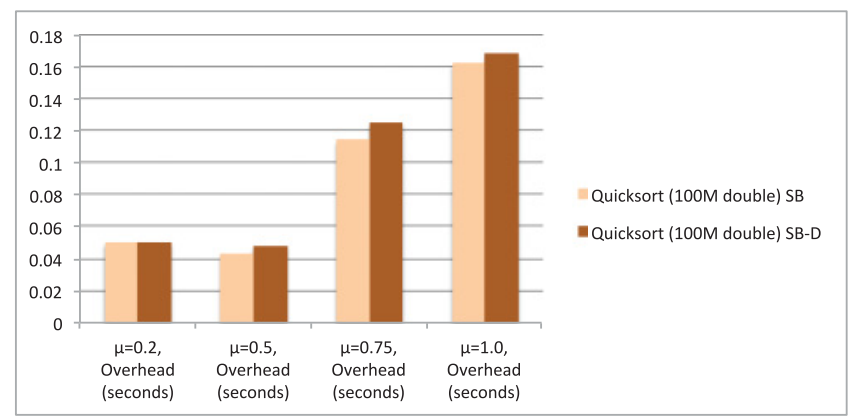

Fig. 11. Overhead of quicksort for $\mu=0.2,0.5,0.75,1.0$ at full bandwidth.

scheduling overheads for six of the algorithms when $\mu=0.2$ and when $\mu=1$. Note that $\mu=1$ depicts the case without our added $\mu$ parameter. The figure shows that the $\mu=0.2$ case reduces the overheads by up to a factor of 3 . Figure 11 shows the sensitivity of quicksort's overhead to various settings of $\mu$. While for this particular algorithm, $\mu=0.5$ is the best of these settings, we have found $\mu=0.2$ to be a good choice when considering all of the algorithms in our study.

Load Balance and the Dilation Parameter $\sigma$. The choice of $\sigma$, determining which tasks are maximal, is an important parameter affecting the performance of spacebounded schedulers, especially their ability to load balance. If $\sigma$ were set to 1 , it is likely that one task that is about the size of the cache gets anchored to the cache, leaving little room for other tasks or strands. This adversely affects load balance, and we would expect to see greater empty queue times. On the other hand, if $\sigma$ were set to a lower value like 0.5, then each cache can allow more than one task or strand to be simultaneously anchored, leading to better load balance. Figure 12 gives an example algorithm (QuickHull) demonstrating this. Figure 13, on the other hand, gives an example algorithm (Cholesky) where increasing $\sigma$ has little impact on empty queue times. In such cases, a larger $\sigma$ allows a larger (fitting) subcomputation to be pinned to a cache, thereby improving cache performance, as shown by the $40 \%$ decrease in L3 cache misses.

Note that if $\sigma$ were set too low (closer to 0), then the number of levels of recursion until the space-bounded schedulers preserve locality would increase, resulting in less effective use of shared caches. 


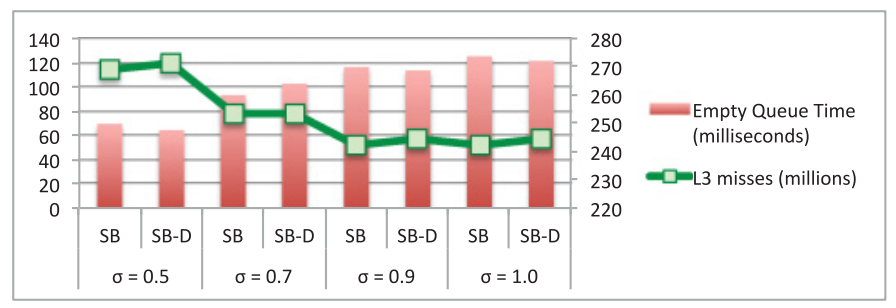

Fig. 12. Empty queue times for QuickHull, varying $\sigma$.

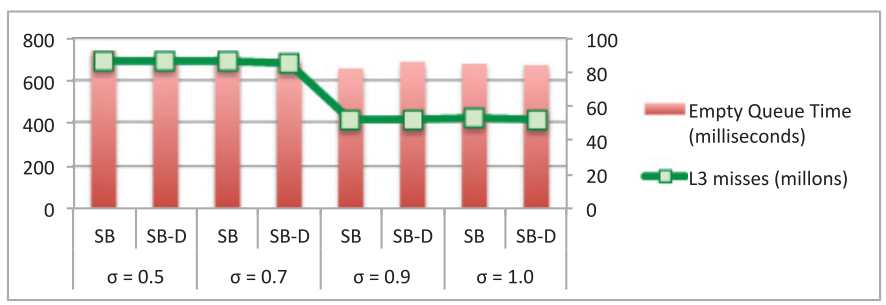

Fig. 13. Empty queue times and L3 cache misses for Cholesky, varying $\sigma$.

Comparison of Scheduler Variants. Looking at the results, we find that while PWS can reduce the number of cache misses by up to $10 \%$ compared to standard WS, it has negligible impact on running times for the benchmarks studied. Similarly, while SB-D is designed to remove a serial bottleneck in the SB scheduler, the runtime (and cache miss) performance of the two are nearly identical. This is because our benchmarks call the scheduler sufficiently infrequently so that the performance difference of each invocation is not noticeable in the overall running time.

\section{CONCLUSION}

We developed a framework for comparing schedulers and deployed it on a 32-core machine with three levels of caches. We used it to compare four schedulers, two each of work-stealing and space-bounded types. As predicted by theory, we did notice that space-bounded schedulers demonstrate some, or even significant, improvement over work-stealing schedulers in terms of cache miss counts on shared caches for most benchmarks. In memory-intensive benchmarks with low instruction-count-to-cachemiss-count ratios, an improvement in L3 miss count because of space-bounded schedulers can improve running time, despite their added overheads. On the other hand, for compute-intensive benchmarks or benchmarks with highly optimized cache complexities, work-stealing schedulers are slightly faster, because of their low scheduling overhead. Improving the overhead of space-bounded schedulers further could make the case for space-bounded schedulers stronger and is an important direction for future work.

Our experiments were run on an Intel ${ }^{\circledR}$ multicore with (only) eight cores per socket, 32 cores total, and one level of shared cache (the L3). The experiments make it clear that as the core count per socket goes up (as is expected with each new generation), the advantages of space-bounded schedulers should increase due to the increased benefit of avoiding cache conflicts among the many unrelated threads sharing the limited on-chip cache capacity. As the core count per socket goes up, the available bandwidth per core tends to decrease, again increasing space-bounded schedulers' advantage. We also anticipate a greater advantage for space-bounded schedulers over work-stealing schedulers when more cache levels are shared and when the caches are shared among a greater number of cores. Such studies are left to future work, when such multicores 
become available. On the other hand, compute-intensive benchmarks will likely continue to benefit from the lower scheduling overheads of work-stealing schedulers for the next few generations of multicores, if not longer.

\section{APPENDIX}

\section{A. ILLUSTRATION OF THE PROGRAMMING INTERFACE}

Figure 14 depicts the quicksort algorithm described in Section 5.1 implemented in the interface described in Section 3.1. Not all subroutines are shown. The algorithm is

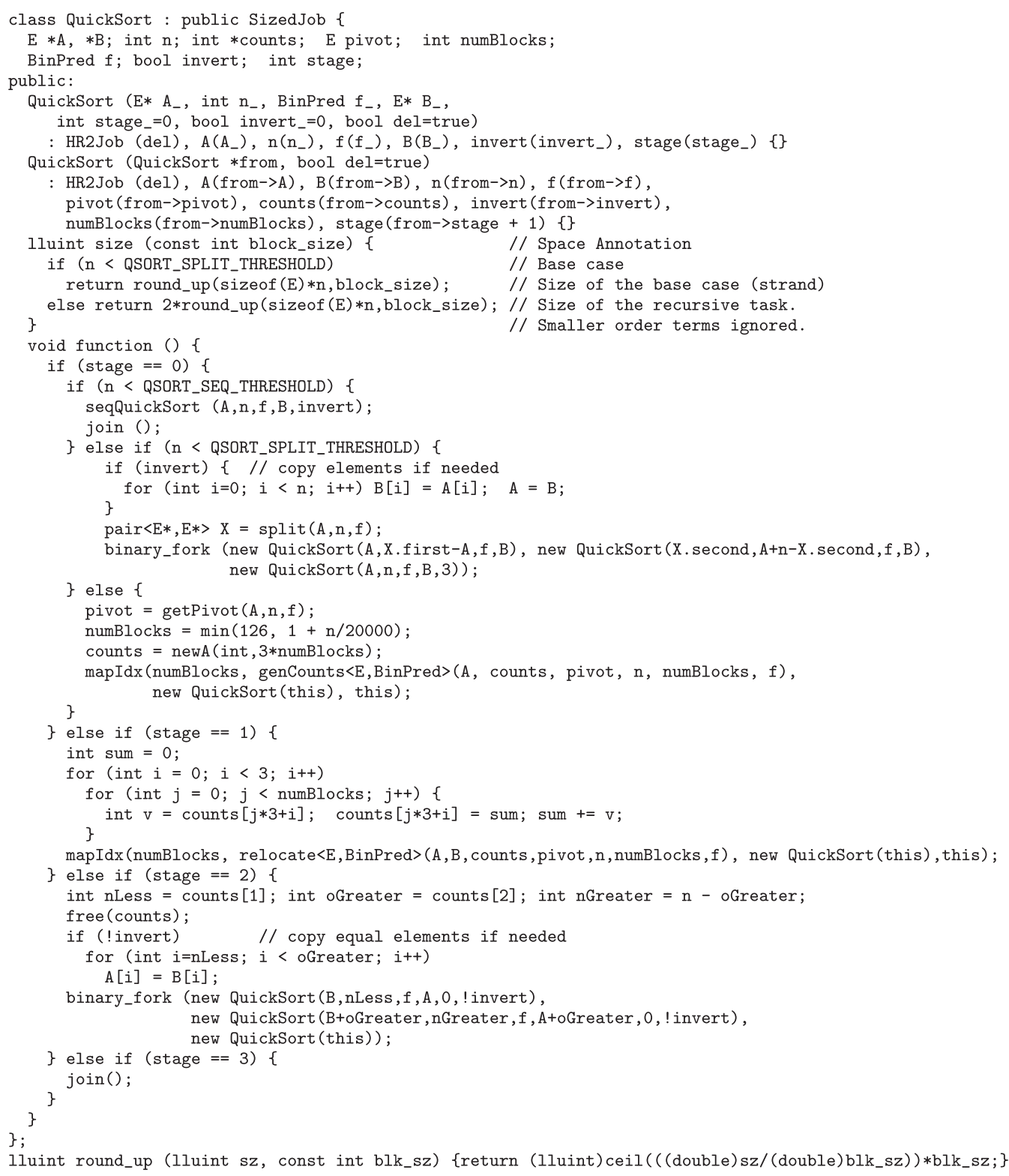

Fig. 14. QuickSort algorithm with space annotation. 


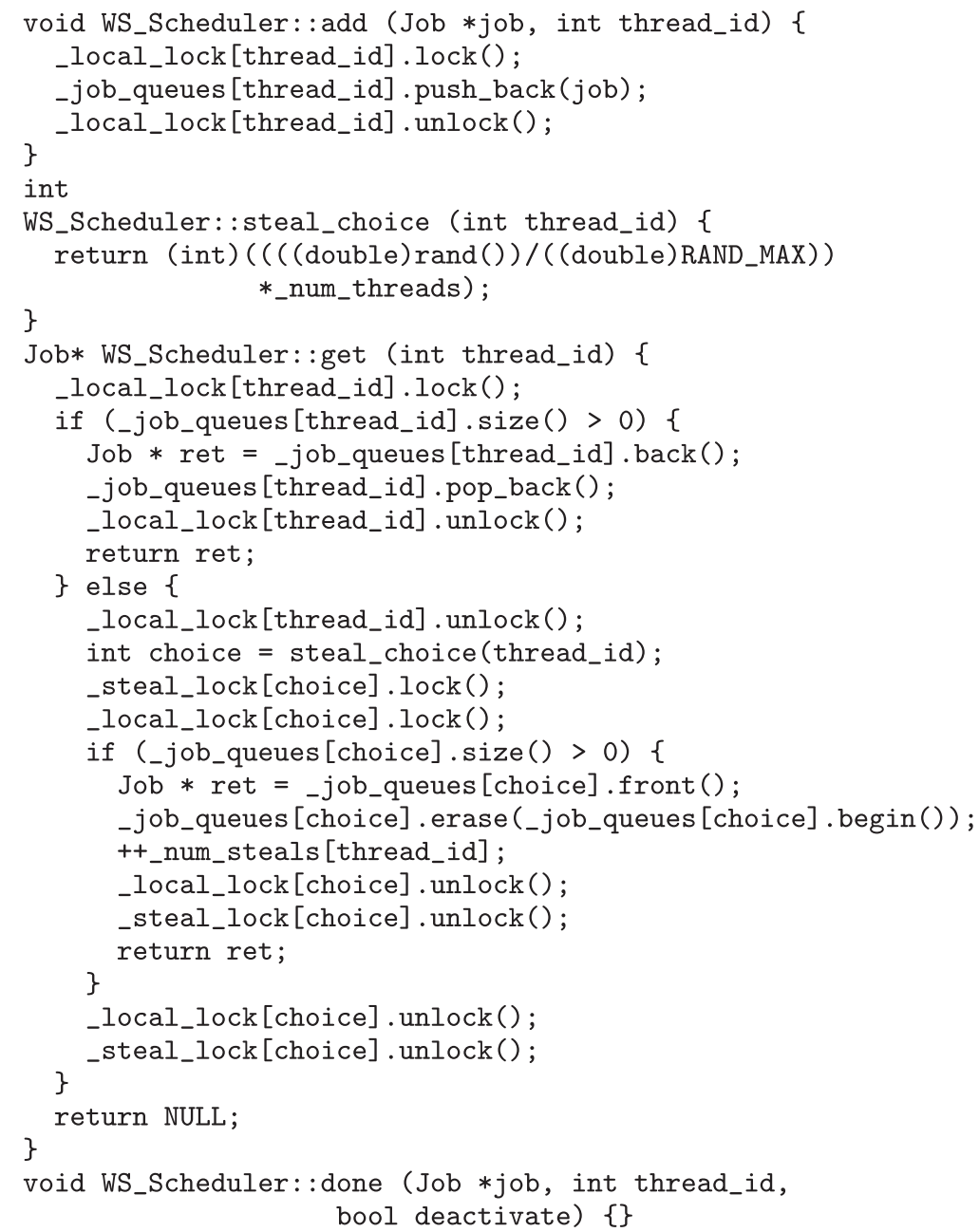

Fig. 15. WS scheduler implemented in scheduler interface.

implemented as a derived class of the SizedJob class, which in turn is derived from Job. An instance of QuickSort class constitutes a task, and the size method returns the space footprint of the task.

\section{B. WORK-STEALING SCHEDULER}

Figure 15 provides an example of a work-stealing scheduler implemented using the scheduler interface presented in Section 3.1. The Job* argument passed to the add and done functions may be instances of one of the derived classes of Job* that carry additional information helpful to the scheduler.

\section{MEASURING HARDWARE COUNTERS}

Multicore processors based on newer architectures like Intel ${ }^{\circledR}$ Nehalem-EX and Sandybridge contain numerous functional components such as cores (which includes the CPU and lower-level caches), DRAM controllers, bridges to the intersocket interconnect (QPI), and higher-level cache units (L3). Each component is provided with a 


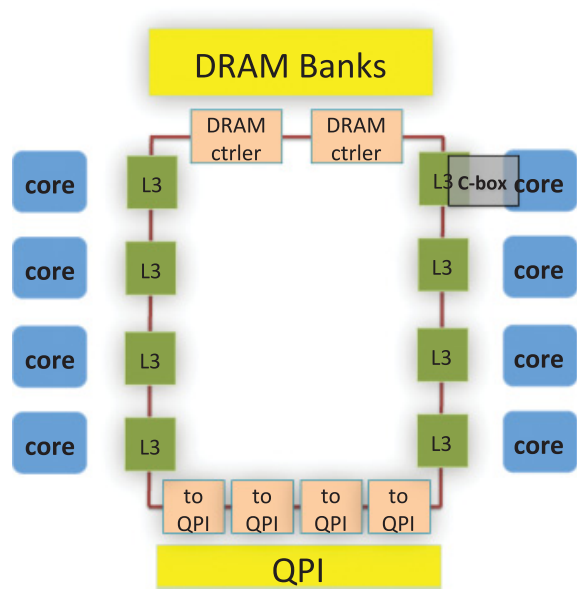

Fig. 16. Layout of eight cores and L3 cache banks on a bidirectional ring in Xeon ${ }^{\circledR} 7560$. Each L3 bank hosts a performance-monitoring unit called C-box that measures traffic into and out of the L3 bank.

performance-monitoring unit (PMU) - a collection of hardware registers that can track statistics of events relevant to the component.

For instance, while the core PMU on Xeon ${ }^{\circledR} 7500$ series (our experimental setup, see Figure 1(a)) is capable of providing statistics such as the number of instructions, L1 and L2 cache hit/miss statistics, and traffic going in and out, it is unable to monitor L3 cache misses (which constitute a significant portion of active time). This is because L3 cache is a separate unit with its own PMU(s). In fact, each Xeon ${ }^{\circledR} 7560$ die has eight L3 cache banks on a bus that also connects DRAM and QPI controllers (see Figure 16). Each L3 bank is connected to a core via buffered queues. The address space is hashed onto the L3 banks so that a unique bank is responsible for each address. To collect L3 statistics such as L3 misses, we monitor PMUs (called C-Boxes on Nehalem-EX) on all L3 banks and aggregate the numbers in our results.

Software access to core PMUs on most Intel ${ }^{\circledR}$ architectures is well supported by several tools including the Linux kernel, the Linux perf tool, and higher-level APIs such as libpfm [Perfmon2 2012]. We use the libpfm library to provide fine-grained access to the core PMU. However, access to uncore PMUs-complex architecture-specific components like the C-Box-is not supported by most tools. Newer Linux kernels (3.7+) are incrementally adding software interfaces to these PMUs at the time of this writing, but we are only able to make program-wide measurements using this interface rather than fine-grained measurements. For accessing uncore counters, we adapt the Intel ${ }^{\circledR}$ PCM 2.4 tool [Intel 2013b].

To count L3 cache misses, the uncore counters in the C-boxes were programmed using the Intel ${ }^{\circledR}$ PCM tool to count misses that occur due to any reason (LLC_MISSES - event code: 0x14, umask: 0b111) and L3 cache fills of missing cache lines in any coherence state (LLC_S_FILLS - event code: 0x16, umask: 0b1111). Since the two counts are complementary-one counts number of missing lines and other the number of missing lines fetched and filled-we would expect them to be the same. Indeed, both the numbers concur up to three significant digits in most cases. Therefore, only the L3 cache miss numbers are reported in this article.

\section{REFERENCES}

Umut A. Acar, Guy E. Blelloch, and Robert D. Blumofe. 2002. The data locality of work stealing. Theory Comp. Sys. 35, 3 (2002), 321-347. 
Bowen Alpern, Larry Carter, and Jeanne Ferrante. 1993. Modeling parallel computers as memory hierarchies. In Proceedings of the 1993 Conference on Programming Models for Massively Parallel Computers. IEEE, 116-123.

Guy E. Blelloch, Rezaul A. Chowdhury, Phillip B. Gibbons, Vijaya Ramachandran, Shimin Chen, and Michael Kozuch. 2008. Provably good multicore cache performance for divide-and-conquer algorithms. In Proceedings of the 19th Annual ACM-SIAM Symposium on Discrete Algorithms (SODA'08). SIAM, Philadelphia, PA, 501-510.

Guy E. Blelloch, Jeremy T. Fineman, Phillip B. Gibbons, and Julian Shun. 2012. Internally deterministic parallel algorithms can be fast. In Proceedings of the 17th ACM SIGPLAN Symposium on Principles and Practice of Parallel Programming (PPoPP'12). ACM, New York, NY, 181-192.

Guy E. Blelloch, Jeremy T. Fineman, Phillip B. Gibbons, and Harsha Vardhan Simhadri. 2011. Scheduling irregular parallel computations on hierarchical caches. In Proceedings of the 23rd Annual ACM Symposium on Parallelism in Algorithms and Architectures (SPAA'11). ACM, New York, NY, 355-366.

Guy E. Blelloch, Jeremy T. Fineman, Phillip B. Gibbons, and Harsha Vardhan Simhadri. 2013. Programcentric cost models for locality. In ACM SIGPLAN Workshop on Memory Systems Performance and Correctness (MSPC'13). ACM, New York, NY, Article 6, 2 pages.

Guy E. Blelloch, Phillip B. Gibbons, and Yossi Matias. 1999. Provably efficient scheduling for languages with fine-grained parallelism. J. ACM 46, 2 (March 1999), 281-321.

Guy E. Blelloch, Phillip B. Gibbons, and Harsha Vardhan Simhadri. 2010. Low-depth cache oblivious algorithms. In Proceedings of the 22th Annual Symposium on Parallelism in Algorithms and Architectures (SPAA'10). ACM, New York, NY, 189-199.

Robert D. Blumofe, Matteo Frigo, Christopher F. Joerg, Charles E. Leiserson, and Keith H. Randall. 1996. An analysis of dag-consistent distributed shared-memory algorithms. In Proceedings of the 8th Annual ACM Symposium on Parallel Algorithms and Architectures (SPAA'96). ACM, New York, NY, 297-308.

Robert D. Blumofe and Charles E. Leiserson. 1999. Scheduling multithreaded computations by work stealing. J. ACM 46, 5 (Sept. 1999), 720-748.

Rezaul Alam Chowdhury and Vijaya Ramachandran. 2007. The cache-oblivious gaussian elimination paradigm: Theoretical framework, parallelization and experimental evaluation. In Proceedings of the 19th Annual ACM Symposium on Parallel Algorithms and Architectures (SPAA'07). ACM, New York, NY, 71-80.

Rezaul Alam Chowdhury and Vijaya Ramachandran. 2008. Cache-efficient dynamic programming algorithms for multicores. In Proceedings of the 20th ACM Symposium on Parallelism in Algorithms and Architectures (SPAA'08). ACM, New York, NY, 207-216.

Rezaul Alam Chowdhury, Vijaya Ramachandran, Francesco Silvestri, and Brandon Blakeley. 2013. Oblivious algorithms for multicores and networks of processors. J. Parallel Distrib. Comput. 73, 7 (2013), 911-925. Best Papers of IPDPS 2010, 2011 and 2012.

Rezaul Alam Chowdhury, Francesco Silvestri, Brandon Blakeley, and Vijaya Ramachandran. 2010. Oblivious algorithms for multicores and network of processors. In Proceedings of the 24th International Parallel and Distributed Processing Symposium. IEEE Computer Society, 1-12.

Kayvon Fatahalian, Daniel Reiter Horn, Timothy J. Knight, Larkhoon Leem, Mike Houston, Ji Young Park, Mattan Erez, Manman Ren, Alex Aiken, William J. Dally, and Pat Hanrahan. 2006. Sequoia: Programming the memory hierarchy. In Proceedings of the 2006 ACM/IEEE Conference on Supercomputing (SC'06). ACM, New York, NY, Article 83, 13 pages.

Matteo Frigo, Charles E. Leiserson, and Keith H. Randall. 1998. The implementation of the Cilk-5 multithreaded language. In Proceedings of the ACM SIGPLAN'98 Conference on Programming Language Design and Implementation (PLDI'98). ACM, 212-223. Proceedings published ACM SIGPLAN Notices, Vol. 33, No. 5, May, 1998.

Fujitsu Technology Solutions. 2010. White Paper: Fujitsu Primergy servers memory performance of Xeon 7500 (Nehalem-EX) based systems. http://globalsp.ts.fujitsu.com/dmsp/Publications/public/ wp-nehalem-ex-memory-performance-ww-en.pdf. (2010).

Intel. 2013a. Intel Thread Building Blocks Reference Manual. http://software.intel.com/sites/products/ documentation/doclib/tbb_sa/help/index.htm\#reference/reference.htm. (2013). Version 4.1.

Intel. 2013b. Performance Counter Monitor (PCM). http://www.intel.com/software/pcm. (2013). Version 2.4.

Andi Kleen. 2004. An NUMA API for Linux. http://halobates.de/numaapi3.pdf. (August 2004).

Ronald Kriemann. 2004. Implementation and Usage of a Thread Pool based on POSIX Threads. http://www. hlnum.org/english/projects/tools/threadpool/doc.html. (2004).

Doug Lea. 2000. A java fork/join framework. In ACM Java Grande. ACM, New York, NY, 36-43.

Charles E. Leiserson. 2010. The Cilk++ concurrency platform. J. Supercomput. 51, 3 (2010), $244-257$. 
Adam Litke, Eric Mundon, and Nishanth Aravamudan. 2006. libhugetlbfs. http://libhugetlbfs.sourceforge. net. (2006).

Microsoft. 2013. Task Parallel Library. http://msdn.microsoft.com/en-us/library/dd460717.aspx. (2013). NET version 4.5 .

Daniel Molka, Daniel Hackenberg, Robert Schone, and Matthias S. Muller. 2009. Memory performance and cache coherency effects on an Intel Nehalem multiprocessor system. In Proceedings of the 18th International Conference on Parallel Architectures and Compilation Techniques (PACT'09). IEEE, 261270.

Girija J. Narlikar. 2002. Scheduling threads for low space requirement and good locality. Theory Comput. Syst. 35, 2 (2002), 151-187.

OpenMP Architecture Review Board. 2008. OpenMP API. http://www.openmp.org/mp-documents/spec30.pdf. (May 2008). v 3.0.

Perfmon2. 2012. libpfm. http://perfmon2.sourceforge.net/. (2012).

Jean-Noël Quintin and Frédéric Wagner. 2010. Hierarchical work-stealing. In EuroPar. Springer-Verlag, Berlin, 217-229.

Julian Shun, Guy E. Blelloch, Jeremy T. Fineman, Phillip B. Gibbons, Aapo Kyrola, Harsha Vardhan Simhadri, and Kanat Tangwongsan. 2012. Brief announcement: The problem based benchmark suite. www.cs.cmu.edu/ pbbs. In Proceedings of the 24th ACM Symposium on Parallelism in Algorithms and Architectures (SPAA'12). ACM, New York, NY, 68-70.

Harsha Vardhan Simhadri. 2013. Program-Centric Cost Models for Locality and Parallelism. Ph.D. Dissertation. CMU. http://reports-archive.adm.cs.cmu.edu/anon/2013/CMU-CS-13-124.pdf.

Daniel Spoonhower, Guy E. Blelloch, Phillip B. Gibbons, and Robert Harper. 2009. Beyond nested parallelism: Tight bounds on work-stealing overheads for parallel futures. In Proceedings of the 21st Annual Symposium on Parallelism in Algorithms and Architectures (SPAA'09). ACM, New York, NY, 91-100.

Yuan Tang, Ronghui You, Haibin Kan, Jesmin Jahan Tithi, Pramod Ganapathi, and Rezaul A. Chowdhury. 2015. Cache-oblivious wavefront: Improving parallelism of recursive dynamic programming algorithms without losing cache-efficiency. In Proceedings of the 20th ACM SIGPLAN Symposium on Principles and Practice of Parallel Programming (PPoPP'15). ACM, New York, NY, 205-214.

Leslie G. Valiant. 2011. A bridging model for multi-core computing. J. Comput. Syst. Sci. 77, 1 (2011), 154-166.

Received September 2014; revised March 2015; accepted February 2016 